Research Article

\title{
Study of $B_{s} \longrightarrow \phi \ell^{+} \ell^{-}$Decays in the PQCD Factorization Approach with Lattice QCD Input
}

\author{
Su-Ping Jin $\mathbb{D}^{1}$ and Zhen-Jun Xiao $\mathbb{D D}^{1,2}$ \\ ${ }^{1}$ Department of Physics and Institute of Theoretical Physics, Nanjing Normal University, Nanjing, Jiangsu 210023, China \\ ${ }^{2}$ Jiangsu Key Laboratory for Numerical Simulation of Large Scale Complex Systems, Nanjing Normal University, \\ Nanjing 210023, China
}

Correspondence should be addressed to Zhen-Jun Xiao; xiaozhenjun@njnu.edu.cn

Received 29 May 2021; Revised 29 August 2021; Accepted 13 September 2021; Published 9 November 2021

Academic Editor: Andrea Coccaro

Copyright (C) 2021 Su-Ping Jin and Zhen-Jun Xiao. This is an open access article distributed under the Creative Commons Attribution License, which permits unrestricted use, distribution, and reproduction in any medium, provided the original work is properly cited. The publication of this article was funded by $\mathrm{SCOAP}^{3}$.

In this paper, we studied systematically the semileptonic decays $B_{s} \longrightarrow \phi l^{+} l^{-}$with $l^{-}=\left(e^{-}, \mu^{-}, \tau^{-}\right)$by using the perturbative QCD (PQCD) and the "PQCD+Lattice" factorization approach, respectively. We first evaluated all relevant form factors $F_{i}\left(q^{2}\right)$ in the low- $q^{2}$ region using the PQCD approach, and we also took the available lattice QCD results at the high- $q^{2}$ region as additional input to improve the extrapolation of $F_{i}\left(q^{2}\right)$ from the low- $q^{2}$ region to the endpoint $q_{\max }^{2}$. We then calculated the branching ratios and many other physical observables $A_{\mathrm{FB}}^{l}, F_{L}^{\phi}, S_{3,4,7}$, and $A_{5,6,8,9}$ and the clean angular observables $P_{1,2,3}$ and $P_{4,5,6,8}$. From our studies, we find the following points: (a) the PQCD and "PQCD+Lattice" predictions of $\mathscr{B}\left(B_{s} \longrightarrow \phi \mu^{+} \mu^{-}\right)$are about $7 \times 10^{-7}$, which agree well with the LHCb measured values and the QCD sum rule prediction within still large errors; (b) we defined and calculated the ratios of the branching ratios $R_{\phi}^{e \mu}$ and $R_{\phi}^{\mu \tau}$; (c) the PQCD and "PQCD+Lattice" predictions of the longitudinal polarization $F_{L}$, the $\mathrm{CP}$-averaged angular coefficients $S_{3,4,7}$, and the $\mathrm{CP}$ asymmetry angular coefficients $A_{5,6,8,9}$ agree with the LHCb measurements in all considered bins within the still large experimental errors; and (d) for those currently still unknown observables $R_{\phi}^{e \mu}, R_{\phi}^{\mu \tau}, A_{\mathrm{FB}}^{l}, P_{1,2,3}$, and $P_{4,5,6,8}$, we suggest LHCb and Belle-II Collaboration to measure them in their experiments.

\section{Introduction}

In the standard model (SM) of particle physics, one treats these three generations of the charged leptons $\ell^{-}=\left(e^{-}, \mu^{-}\right.$, $\left.\tau^{-}\right)$as exact copies of each other. These charged leptons behave in the same way but differ only in the masses determined by their Yukawa coupling to the Higgs boson. The lepton flavor universality (LFU), i.e., the equality of the coupling to the all electroweak gauge bosons among three families of leptons, has been regarded as an exact symmetry for quite a long time [1]. In recent years, however, some physics observables associated with the flavor-changing neutral current $(\mathrm{FCNC})$ transitions $b \longrightarrow$ sll have exhibited deviations from the SM expectations. These include the LFU-violating(LFUV) ratios $R_{K}$ and $R_{K^{*}}[2,3]$, whose measurements deviate from $\mu$-e universality [4-6] by around 2.5 $\sigma$. More notably, the measurements of the angular observable $P_{5}^{\prime}$ of $B \longrightarrow K^{*} \mu^{+} \mu^{-}$decay in the large recoil region [7-11] as reported by the $\mathrm{LHCb}[12,13]$ and Belle Collaboration [14] point to a deviation of about $3 \sigma$ with respect to the SM prediction [15].

As is well known, the FCNC $b \longrightarrow s$ transition is forbidden at tree-level, but proceeds by way of loop diagrams with a very low rate. Due to the strong suppression within SM, such kinds of FCNC decays may be sensitive to the possible new physics (NP) effects. Therefore, the semileptonic $b \longrightarrow$ sll decay has received striking attentions by means of measurements of the inclusive $B \longrightarrow X_{s} \ell^{+} \ell^{-}$and/or the exclusive $B \longrightarrow K^{(*)} \ell^{+} \ell^{-}$decays and their comparison with the SM predictions. Besides the decay rates, many 
angular observables of the semileptonic $B \longrightarrow K^{*} \mu^{+} \mu^{-}$ decays have also been measured previously [12-14]. The precision of the experimental measurements will also be expected to upgrade remarkably in the forthcoming year.

The semileptonic decay $B_{s} \longrightarrow \phi \mu^{+} \mu^{-}$, which is closely relevant to the decay $B \longrightarrow K^{*} \mu^{+} \mu^{-}$, offers an alternative scene to check out the same fundamental quark process, in a different hadronic background. On the theoretical side, various studies on the quark level $b \longrightarrow s$ transition and the exclusive $B_{(s)} \longrightarrow V \ell^{+} \ell^{-}$decays by using rather different theories or models have been performed within the SM, such as the constituent quark model or covariant quark model $[16,17]$, the light front quark model [18], the QCD factorization (QCDF) [19], and the light-cone sum rule (LCSR) [20-25], and beyond the SM, such as the universal extra dimension [26, 27] and the supersymmetric theory [28]. On the experimental side, the $B_{s} \longrightarrow \phi \mu^{+} \mu^{-}$decay mode was first observed and studied by the CDF Collaboration [29] and subsequently by the LHCb Collaboration [30-34]. Beyond the measurement of the branching ratio, a rich phenomenology of various kinematical distributions can be presented. While the angular distributions were found to be consistent with the SM expectations obtained in References $[22,23]$, however, LHCb also observed a deficit with respect to the SM prediction for the branching ratio $B_{s}^{0} \longrightarrow$ $\phi \mu^{+} \mu^{-}$in the low- $q^{2}$ region: the tension between the theory and experiment is about $3 \sigma$ in the region $1.0 \leq q^{2} \leq 6.0 \mathrm{Ge}$ $\mathrm{V}^{2}$, where the form factors are evaluated by using the combined fit of lattice and the LCSR results [22, 23].

In a previous paper [35], the semileptonic $B_{s} \longrightarrow K^{(*)} \ell^{+}$ $\ell^{+}$decays have been studied by us using the perturbative QCD (PQCD) factorization approach [36-47]. In this paper, we will make systematic studies for the semileptonic $B_{s} \longrightarrow$ $\phi \ell^{+} \ell^{-}$and present the theoretical predictions for many physical observables:

(1) For $B_{s} \longrightarrow \phi \ell^{+} \ell^{-}$decays, we treat them as a fourbody decay $B_{s} \longrightarrow \phi\left(\longrightarrow K^{-} K^{+}\right) \ell^{+} \ell^{-}$described by four kinematic variables: the lepton invariant mass squared $q^{2}$ and three angles $\left(\theta_{K}, \theta_{\ell}, \Phi\right)$. We defined and calculated the full angular decay distribution, the transverse amplitudes, the partially integrated decay amplitudes over the angles $\left(\theta_{K}, \theta_{\ell}, \Phi\right)$, the CPaveraged differential branching, the ratios $R^{e \mu}(\phi)$ and $R^{\mu \tau}(\phi)$ of the branching ratios, the forwardbackward asymmetry $A_{\mathrm{FB}}\left(q^{2}\right)$, the $\phi$ polarization fraction $F_{L}\left(q^{2}\right)$, the CP-averaged (asymmetry) angular coefficients $S_{i}\left(A_{i}\right)$, and the optimized observables $P_{i}$ and $P_{i}^{\prime}$. Following Reference [48], where the authors approved that the possible S-wave correction to the branching fractions of $B_{s} \longrightarrow \phi\left(\longrightarrow K^{-} K^{+}\right) \ell^{+} \ell^{-}$ decays is small and may modify the differential decay widths by about $5 \%$ only, we therefore will take the Swave correction to the branching fractions as an additional uncertainty of $5 \%$ in magnitude

(2) We used both the PQCD factorization approach and the "PQCD+Lattice" approach to determine the values and their $q^{2}$-dependence of the $B_{s} \longrightarrow \phi$ transition form factors. We used the $z$-series parametrization to make the extrapolation for all form factors from the low- $q^{2}$ region to the endpoint $q_{\max }^{2}$. We will calculate the branching ratios and all other physical observables by using the PQCD approach itself and the "PQCD +Lattice" approach, respectively, and compare their predictions with those currently available experimental measurements

The paper is organized as follows: In Section 2, we give a short review for the kinematics of the $B_{s} \longrightarrow \phi \ell^{+} \ell^{-}$decays including distribution amplitudes of $B_{s}$ and $\phi$ mesons and the effective Hamiltonian for the quark level $b \longrightarrow s \ell^{+} \ell^{-}$. In Section 3, we define explicitly all physical observables for $B_{s} \longrightarrow \phi \ell^{+} \ell^{-}$decays. In Section 4 , we present our theoretical predictions of all relevant physical observables of the considered decay modes, compare these predictions with those currently available experimental measurements, and make some phenomenological analysis. A short summary is given in the last section.

\section{Kinematics and Theoretical Framework}

2.1. Kinematics and Wave Functions. We treat the $B_{s}$ meson at rest as a heavy-light system. The kinematics of the semileptonic $B_{s} \longrightarrow \phi \ell^{+} \ell^{-}$decays in the large-recoil (low- $q^{2}$ ) region will be discussed below, where the PQCD factorization approach is applicable to the considered decays. In the rest frame of $\bar{B}_{s}^{0}$ meson, we define the $\bar{B}_{s}^{0}$ meson momentum $p_{1}$ and the $\phi$ momentum $p_{2}$ in the light-cone coordinates as Reference [41]. We also use $x_{i}$ to denote the momentum fraction of light antiquark in each meson and set the momentum $p_{i}$ and $k_{i}$ (the momenta carried by the spectator quark in $B_{s}$ and $\phi$ meson) in the following forms:

$$
\begin{aligned}
& p_{1}=\frac{m_{B_{s}}}{\sqrt{2}}\left(1,1,0_{\perp}\right), \\
& p_{2}=\frac{r m_{B_{s}}}{\sqrt{2}}\left(\eta^{+}, \eta^{-}, 0_{\perp}\right), \\
& k_{1}=\left(0, x_{1} \frac{m_{B_{s}}}{\sqrt{2}}, k_{1 \perp}\right), \\
& k_{2}=\frac{m_{B_{s}}}{\sqrt{2}}\left(x_{2} r \eta^{+}, x_{2} r \eta^{-}, k_{2 \perp}\right),
\end{aligned}
$$

where the mass ratio $r=m_{\phi} / m_{B_{s}}$ and the factor $\eta^{ \pm}$is defined in the following form:

$$
\eta^{ \pm}=\eta \pm \sqrt{\eta^{2}-1}, \text { with } \eta=\frac{1}{2 r}\left[1+r^{2}-\frac{q^{2}}{m_{B_{s}}^{2}}\right]
$$

where $q=p_{1}-p_{2}$ is the lepton-pair four-momentum. For the final state $\phi$ meson, its longitudinal and transverse 
polarization vector $\varepsilon_{L, T}$ can be written in the form of $\varepsilon_{L}=($ $\left.\eta^{+},-\eta^{-}, 0_{\perp}\right) / \sqrt{2}$ and $\varepsilon_{T}=(0,0,1)$.

For the $B_{s}$ meson wave function, we use the same kind of parameterizations as in References [42-44].

$$
\Phi_{B_{s}}=\frac{i}{\sqrt{2 N_{c}}}\left(\not p_{B_{s}}+m_{B_{s}}\right) \gamma_{5} \phi_{B_{s}}\left(k_{1}\right)
$$

Here, only the contribution of the Lorentz structure $\phi_{B_{s}}$ $\left(k_{1}\right)$ is taken into account, since the contribution of the second Lorentz structure $\bar{\phi}_{B_{s}}$ is numerically small $[49,50]$ and has been neglected. We adopted the distribution amplitude of the $B_{s}$ meson in the similar form as that of $B$-meson in the $S U(3)_{f}$ limit being widely used in the PQCD approach [42-44]

$$
\phi_{B_{s}}(x, b)=N_{B_{s}} x^{2}(1-x)^{2} \exp \left[-\frac{m_{B_{s}}^{2} x^{2}}{2 \omega_{B_{s}}^{2}}-\frac{1}{2}\left(\omega_{B_{s}} b\right)^{2}\right] .
$$

In order to estimate the theoretical uncertainties induced by the variations of $\phi_{B_{s}}(x, b)$, one usually take $\omega_{B_{s}}=0.50 \pm$ $0.05 \mathrm{GeV}$ for $B_{s}^{0}$ meson $[38,42]$. The normalization factor $N_{B_{s}}$ depends on the values of the shape parameter $\omega_{B_{s}}$ and the decay constant $f_{B_{s}}$ and defined through the normalization relation: $\int_{0}^{1} d x \phi_{B_{s}}(x, 0)=f_{B_{s}} /(2 \sqrt{6})[42,44]$.

For the vector meson $\phi$, the longitudinal and transverse polarization components can both provide the contribution. Here, we adopt the wave functions of the vector $\phi$ as in Reference [42]:

$$
\begin{aligned}
& \Phi_{\phi}^{\|}\left(p, \varepsilon_{L}\right)=\frac{i}{\sqrt{6}}\left[\phi_{L} m_{\phi} \phi_{\phi}(x)+\phi_{L} \not \not \phi_{\phi}^{t}(x)+m_{\phi} \phi_{\phi}^{s}(x)\right] \\
& \Phi_{\phi}^{\perp}\left(p, \varepsilon_{T}\right)=\frac{i}{\sqrt{6}}\left[\phi_{T} m_{\phi} \phi_{\phi}^{v}(x)+\phi_{T} \not \not \phi_{\phi}^{T}(x)+m_{\phi} \varepsilon_{\omega \nu \rho \sigma} \gamma_{5} \gamma^{\omega} \varepsilon_{T}^{v} n^{\rho} v^{\sigma} \phi_{\phi}^{a}(x)\right]
\end{aligned}
$$

where $p$ and $m_{\phi}$ are the momentum and the mass of the $\phi$ meson, $\varepsilon_{L}$ and $\varepsilon_{T}$ correspond to the longitudinal and transverse polarization vectors of the vector meson $\phi$, respectively. The twist-2 DAs $\phi_{\phi}$ and $\phi_{\phi}^{T}$ in Equations (5) and (6) can be reconstructed as a Gegenbauer expansion [42]:

$$
\begin{aligned}
& \phi_{\phi}(x)=\frac{3 f_{\phi}}{\sqrt{6}} x(1-x)\left[1+\sum_{n=1}^{2} a_{n \phi}^{\|} C_{n}^{3 / 2}(t)\right], \\
& \phi_{\phi}^{T}(x)=\frac{3 f_{\phi}^{T}}{\sqrt{6}} x(1-x)\left[1+\sum_{n=1}^{2} a_{n \phi}^{\perp} C_{n}^{3 / 2}(t)\right],
\end{aligned}
$$

where $t=2 x-1$ and $a_{1,2}^{\|, \perp}$ are the Gegenbauer moments, while $C_{1,2}^{3 / 2}$ are the Gegenbauer polynomials as given in Reference [42]. $f_{\phi}$ and $f_{\phi}^{T}$ are the longitudinal and transverse components of the decay constants of the vector meson $\phi$ with $f_{\phi}=0.231 \pm 0.004 \mathrm{GeV}$ and $f_{\phi}^{T}=0.20 \pm 0.01 \mathrm{GeV}$ as given in Reference [42]. For the relevant Gegenbauer moments, we use the same ones as those in References [42, $46,47,51]$.

$$
\begin{aligned}
& a_{1}^{\|, \perp}=0, \\
& a_{2 \phi}^{\|}=0.18 \pm 0.08, \\
& a_{2 \phi}^{\perp}=0.14 \pm 0.07 .
\end{aligned}
$$

The twist-3 DAs $\phi_{\phi}^{s, t}$ and $\phi_{\phi}^{v, a}$ in Equations (5) and (6) are the same ones as those defined in Reference [42]:

$$
\begin{aligned}
\phi_{\phi}^{t} & =\frac{3 f_{\phi}^{T}}{2 \sqrt{6}} t^{2}, \\
\phi_{\phi}^{s} & =\frac{3 f_{\phi}^{T}}{2 \sqrt{6}}(-t), \\
\phi_{\phi}^{v} & =\frac{3 f_{\phi}}{8 \sqrt{6}}\left(1+t^{2}\right), \\
\phi_{\phi}^{a} & =\frac{3 f_{\phi}}{4 \sqrt{6}}(-t),
\end{aligned}
$$

where $t=2 x-1$.

2.2. Effective Hamiltonian for $b \longrightarrow s \ell^{+} \ell^{-}$Decays. The effective Hamiltonian for the considered semileptonic decay $B_{s} \longrightarrow \phi \ell^{+} \ell^{-}$is defined by the same one as in References [52-56]:

$$
\begin{aligned}
\mathscr{H}_{\text {eff }}= & -\frac{4 G_{F}}{\sqrt{2}}\left\{V_{t b} V_{t s}^{*}\left[C_{1}(\mu) \mathcal{O}_{1}^{c}(\mu)+C_{2}(\mu) \mathcal{O}_{2}^{c}(\mu)+\sum_{i=3}^{10} C_{i}(\mu) \mathcal{O}_{i}(\mu)\right]\right. \\
& \left.+V_{u b} V_{u s}^{*}\left[C_{1}(\mu)\left[\mathcal{O}_{1}^{c}(\mu)-\mathcal{O}_{1}^{u}(\mu)\right]+C_{2}(\mu)\left[\mathcal{O}_{2}^{c}(\mu)-\mathcal{O}_{2}^{u}(\mu)\right]\right]\right\}+ \text { h.c., }
\end{aligned}
$$

where $G_{F}=1.16638 \times 10^{-5} \mathrm{GeV}^{-2}$ is the Fermi constant and $V_{i j}$ are the CKM matrix elements. For the operators $\mathcal{O}_{i}$, we adopt those as defined in the so-called $\gamma_{5}$-free basis $[57,58]$. Following Reference [59], the operators $\mathcal{O}_{i}$ can be written in the following form: 


$$
\begin{array}{cc}
\mathcal{O}_{1}^{c}=\left(\bar{s} \gamma_{\mu} T^{a} P_{L} c\right)\left(\bar{c} \gamma_{\mu} T^{a} P_{L} b\right), & \mathcal{O}_{2}^{c}=\left(\bar{s} \gamma_{\mu} P_{L} c\right)\left(\bar{c} \gamma_{\mu} P_{L} b\right), \\
\mathcal{O}_{1}^{u}=\left(\bar{s} \gamma_{\mu} T^{a} P_{L} u\right)\left(\bar{u} \gamma_{\mu} T^{a} P_{L} b\right), & \mathcal{O}_{2}^{u}=\left(\bar{s} \gamma_{\mu} P_{L} u\right)\left(\bar{u} \gamma_{\mu} P_{L} b\right), \\
\mathcal{O}_{3}=\left(\bar{s} \gamma_{\mu} P_{L} b\right) \sum_{q}\left(\bar{q} \gamma_{\mu} q\right), & \mathcal{O}_{4}=\left(\bar{s} \gamma_{\mu} T^{a} P_{L} b\right) \sum_{q}\left(\bar{q} \gamma_{\mu} T^{a} q\right), \\
\mathcal{O}_{5}=\left(\bar{s} \gamma_{\mu} \gamma_{\nu} \gamma_{\rho} P_{L} b\right) \sum_{q}\left(\bar{q} \gamma^{\mu} \gamma^{v} \gamma^{\rho} q\right), & \mathcal{O}_{6}=\left(\bar{s} \gamma_{\mu} \gamma_{\nu} \gamma_{\rho} T^{a} P_{L} b\right) \sum_{q}\left(\bar{q} \gamma^{\mu} \gamma^{v} \gamma^{\rho} T^{a} q\right), \\
\mathcal{O}_{7}=\frac{e}{g^{2}} m_{b}\left(\bar{s} \sigma^{\mu \nu} P_{R} b\right) F_{\mu \nu}, & \mathcal{O}_{8}=\frac{1}{g} m_{b}\left(\bar{s} \sigma^{\mu \nu} T^{a} P_{R} b\right) G_{\mu \nu}^{a}, \\
\mathcal{O}_{9}=\frac{e^{2}}{g^{2}}\left(\bar{s} \gamma_{\mu} P_{L} b\right) \sum_{\ell}\left(\bar{\ell} \gamma^{\mu} \ell\right), & \mathcal{O}_{10}=\frac{e^{2}}{g^{2}}\left(\bar{s} \gamma_{\mu} P_{L} b\right) \sum_{\ell}\left(\bar{\ell} \gamma^{\mu} \gamma_{5} \ell\right),
\end{array}
$$

where $\mathcal{O}_{1,2}^{c, u}$ are the current-current operators, $\mathcal{O}_{3-6}$ are the QCD penguin operators, $\mathcal{O}_{7,8}$ are the electromagnetic and chromomagnetic penguin operators, respectively, and finally, $\mathcal{O}_{9,10}$ are the semileptonic operators. The inclusion of the factors $4 \pi / g^{2}=1 / \alpha_{s}$ in the definition of the operators $\mathcal{O}_{7,8,9,10}$ serves to allow a more transparent organisation of the expansion of the relevant Wilson coefficients as defined in References $[59,60]$ up to next-to-next-to-leading order (NNLO). They are then evolved from the scale $\mu=m_{W}$ down to the scale $\mu=m_{b}$ using the renormalization group equations.

Since the contributions from the subleading chromomagnetic penguin, quark loop, and annihilation diagrams are highly suppressed for the considered $b \longrightarrow s \ell^{+} \ell^{-}$decays [55], we will neglect them in our calculations. Using the effective Hamiltonian in Equation (11), the decay amplitude for $b \longrightarrow s \ell^{+} \ell^{-}$loop transition can be decomposed as a product of a short-distance contributions through Wilson coefficients and long-distance contribution which is further expressed in terms of form factors:

$$
\begin{aligned}
A\left(b \longrightarrow s \ell^{+} \ell^{-}\right)= & \frac{G_{F}}{\sqrt{2}} \frac{\alpha_{\mathrm{em}}}{\pi} V_{t b} V_{t s}^{*}\left\{C_{9}^{\mathrm{eff}}\left(q^{2}\right)\left[\bar{s} \gamma_{\mu} P_{L} b\right]\left[\bar{\ell} \gamma^{\mu} \ell\right]\right. \\
& +C_{10}\left[\bar{s} \gamma_{\mu} P_{L} b\right]\left[\bar{\ell} \gamma^{\mu} \gamma_{5} \ell\right]-2 m_{b} C_{7}^{\mathrm{eff}} \\
& \left.\cdot\left[\bar{s} i \sigma_{\mu \nu} \frac{q^{v}}{q^{2}} P_{R} b\right]\left[\bar{\ell} \gamma^{\mu} \ell\right]\right\}
\end{aligned}
$$

where $C_{7}^{\text {eff }}(\mu)$ and $C_{9}^{\text {eff }}(\mu)$ are the effective Wilson coefficients, defined as in References $[44,61]$.

$$
\begin{gathered}
C_{7}^{\mathrm{eff}}(\mu)=C_{7}(\mu)+C_{b \longrightarrow s \gamma} \stackrel{\prime}{\longrightarrow}(\mu), \\
C_{9}^{\mathrm{eff}}\left(\mu, q^{2}\right)=C_{9}(\mu)+Y_{\text {pert }}\left(q^{2}\right)+Y_{\text {res }}\left(q^{2}\right) .
\end{gathered}
$$

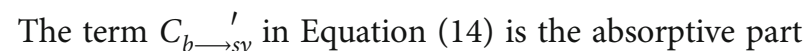
of $b \longrightarrow s \gamma$ transition and was given in Reference [61].

$$
\begin{aligned}
C_{b \longrightarrow s \gamma} \stackrel{\prime}{\hookrightarrow}(\mu)= & i \alpha_{s}\left\{\frac{2}{9} \eta^{14 / 23}\left[\frac{x_{t}\left(x_{t}^{2}-5 x_{t}-2\right)}{8\left(x_{t}-1\right)^{3}}+\frac{3 x_{t}^{2} \ln x_{t}}{4\left(x_{t}-1\right)^{4}}-0.1687\right]\right. \\
& \left.-0.03 C_{2}(\mu)\right\},
\end{aligned}
$$

where $x_{t}=m_{t}^{2} / m_{W}^{2}$ and $\eta=\alpha_{s}\left(m_{W}\right) / \alpha_{s}(\mu)$. The explicit expressions of the term $Y_{\text {pert }}\left(q^{2}\right)$ and $Y_{\text {res }}\left(q^{2}\right)$ in Equation (15) are of the following form [56, 62-65]:

$$
\begin{aligned}
Y_{\text {pert }}\left(q^{2}\right)= & 0.124 \omega(\widehat{s})+g\left(\widehat{m}_{c}, \widehat{s}\right) C_{0}+\lambda_{u}\left[g\left(\widehat{m}_{c}, \widehat{s}\right)\right. \\
& \left.-g\left(\widehat{m}_{u}, \widehat{s}\right)\right]\left(3 C_{1}+C_{2}\right)-\frac{1}{2} g\left(\widehat{m}_{b}, \widehat{s}\right)\left(C_{3}+3 C_{4}\right) \\
& -\frac{1}{2} g\left(\widehat{m}_{b}, \widehat{s}\right)\left(4 C_{3}+4 C_{4}+3 C_{5}+C_{6}\right) \\
& +\frac{2}{9}\left(3 C_{3}+C_{4}+3 C_{5}+C_{6}\right), \\
Y_{\text {res }}\left(q^{2}\right)=- & \frac{3 \pi}{\alpha_{\mathrm{em}}^{2}}\left[C_{0} \cdot \sum_{V=J / \Psi, \Psi^{\prime} \ldots} \frac{m_{V} B\left(V \longrightarrow l^{+} l^{-}\right) \Gamma_{\text {tot }}^{V}}{q_{V}^{2}+i m_{V} \Gamma_{\text {tot }}^{V}}\right. \\
- & \left.\lambda_{u} g\left(\widehat{m}_{u}, \widehat{s}\right)\left(3 C_{1}+C_{2}\right) \cdot \sum_{V=\rho, \omega, \phi} \frac{m_{V} B\left(V \longrightarrow l^{+} l^{-}\right) \Gamma_{\text {tot }}^{V}}{q^{2}-m_{V}^{2}+i m_{V} \Gamma_{\text {tot }}^{V}}\right],
\end{aligned}
$$

where $C_{0}=3 C_{1}+C_{2}+3 C_{3}+C_{4}+3 C_{5}+C_{6}, \widehat{s}=q^{2} / m_{b}^{2}, \widehat{m}_{q}=$ $m_{q} / m_{b}$, and the CKM ratio $\lambda_{u}=V_{u b} V_{u s}^{*} /\left(V_{\mathrm{t} b} V_{t s}^{*}\right)$. In Equation (17), the function $\omega(\widehat{s})$ is the soft-gluon correction to the matrix element of operator $\mathcal{O}_{9}$. The function $g\left(\widehat{m}_{q}, \widehat{s}\right)$ in Equations (17) and (18) is related to the basic fermion loop. The contributions from four quark operators $\mathcal{O}_{1}-\mathcal{O}_{6}$ are usually combined with coefficient $C_{9}$ into an "effective" 
one. One can find the explicit expressions of the function $\omega(\widehat{s})$ and $g\left(\widehat{m}_{q}, \widehat{s}\right)$ easily, for example, in Reference [35] and references therein.

The term $Y_{\text {pert }}\left(q^{2}\right)$ in Equations (15) and (17) defines the short-distance perturbative part that involves the indirect contributions from the matrix element of the four quark operators $\sum_{i=1}^{10}\left\langle\ell^{+} \ell^{-} s\left|\mathcal{O}_{i}\right| b\right\rangle[62-65]$ and lies at the place far away from $c \bar{c}$ resonance regions.

The term $Y_{\text {res }}\left(q^{2}\right)$ in Equations (15) and (18) describes the long-distance resonant contributions related with the $B_{s} \longrightarrow \phi V \longrightarrow \phi\left(V \longrightarrow l^{+} l^{-}\right)$transitions in the resonance regions, where $V=\left(\rho, \omega, \phi, J / \Psi, \psi^{\prime}, \cdots\right)$ are the light vector mesons and $c \bar{c}$ charmonium states. Up to now, the term $Y_{\text {res }}\left(q^{2}\right)$ cannot be calculated from the first principle of QCD and may also introduce the double-counting problem with the term $Y_{\text {pert }}\left(q^{2}\right)$. For more details about such kinds of double-counting problem, one can see the discussions as given in References $[66,67]$. In this paper, we checked the possible effects on the theoretical predictions for the branching ratios and other considered physical observables by including the term $Y_{\text {res }}\left(q^{2}\right)$ or not in our numerical calculations, and we found that the resulted variations of the theoretical predictions are less than $5 \%$. It is much smaller than the total theoretical errors, say around 30-40\%. According to the argument in Reference [68], the term $Y_{\text {res }}\left(q^{2}\right)$ is also generally small. Because of its smallness and the possible double-counting problem, we here simply drop the term $Y_{\text {res }}\left(q^{2}\right)$ out in our numerical evaluations for all physical observables considered in this paper.

2.3. $B_{s} \longrightarrow \phi$ Transition Form Factors. For the vector meson $\phi$ with polarization vector $\varepsilon^{*}$, as usual, the relevant form factors for $B_{s} \longrightarrow \phi$ transitions are $V\left(q^{2}\right)$ and $A_{0,1,2}\left(q^{2}\right)$ of the vector and axial vector currents and $T_{1,2,3}$ of the tensor currents. Between the form factors $A_{0,1,2}\left(q^{2}\right)$ at the point $q^{2}=0$, there is an exact relation $2 m_{\phi} A_{0}(0)=\left(m_{B_{s}}+m_{\phi}\right) A_{1}$ $(0)-\left(m_{B_{s}}-m_{\phi}\right) A_{2}(0)$ in order to avoid the kinematical singularity. Between the form factor $T_{1,2}$, there also exists a relation $T_{1}(0)=T_{2}(0)$ in an algebraic manner which is implied by the identity $\sigma^{\mu \nu} \gamma_{5}=-(i / 2) \varepsilon^{\mu \nu \alpha \beta} \sigma_{\alpha \beta}$ with the $\varepsilon^{0123}=+1$ convention for the Levi-Civita tensor.

Using the well-studied wave functions as given in previous subsection, the PQCD factorization formulas for the relevant form factors of $B_{s} \longrightarrow \phi \ell^{+} \ell^{-}$decays can be calculated and written in the following form:

$$
\begin{aligned}
V\left(q^{2}\right)= & 8 \pi m_{B_{s}}^{2} C_{F}(1+r) \int d x_{1} d x_{2} \int b_{1} d b_{1} b_{2} d b_{2} \phi_{B_{s}}\left(x_{1}\right) \\
& \times\left\{\left[-x_{2} r \phi_{\phi}^{v}\left(x_{2}\right)+\phi_{\phi}^{T}\left(x_{2}\right)+\frac{1+x_{2} r \eta}{\sqrt{\eta^{2}-1}} \phi_{\phi}^{a}\left(x_{2}\right)\right] \cdot H_{1}\left(t_{1}\right)\right. \\
& \left.+\left[\left(r+\frac{x_{1}}{2 \sqrt{\eta^{2}-1}}\right) \phi_{\phi}^{v}\left(x_{2}\right)-\frac{x_{1}-2 r \eta}{2 \sqrt{\eta^{2}-1}} \phi_{\phi}^{a}\left(x_{2}\right)\right] \cdot H_{2}\left(t_{2}\right)\right\},
\end{aligned}
$$

$$
\begin{aligned}
A_{0}\left(q^{2}\right)= & \pi m_{B_{s}}^{2} C_{F} \int d x_{1} d x_{2} \int b_{1} d b_{1} b_{2} d b_{2} \phi_{B_{s}}\left(x_{1}\right) \\
& \times\left\{\left[\left(1+x_{2} r(2 \eta-r)\right) \phi_{\phi}\left(x_{2}\right)+\left(1-2 x_{2}\right) r \phi_{\phi}^{t}\left(x_{2}\right)\right.\right. \\
+ & \left.\frac{(1-r \eta)-2 x_{2} r(\eta-r)}{\sqrt{\eta^{2}-1}} \phi_{\phi}^{s}\left(x_{2}\right)\right] \cdot H_{1}\left(t_{1}\right) \\
+ & {\left[\left[\frac{x_{1}}{\sqrt{\eta^{2}-1}}\left(\frac{\eta+r}{2}-r \eta^{2}\right)+\left(\frac{x_{1}}{2}-x_{1} r \eta+r^{2}\right)\right] \phi_{\phi}\left(x_{2}\right)\right.} \\
& \left.\left.-\left[\frac{x_{1}(1-r \eta)+2 r(r-\eta)}{\sqrt{\eta^{2}-1}}-x_{1} r\right] \phi_{\phi}^{s}\left(x_{2}\right)\right] \cdot H_{2}\left(t_{2}\right)\right\}, \\
A_{1}\left(q^{2}\right)= & 16 \pi m_{B_{s}}^{2} C_{F} \frac{r}{1+r} \int d x_{1} d x_{2} \int b_{1} d b_{1} b_{2} d b_{2} \phi_{B_{s}}\left(x_{1}\right) \\
& \times\left\{\left[\left(1+x_{2} r \eta\right) \phi_{\phi}^{v}\left(x_{2}\right)+\left(\eta-2 x_{2} r\right) \phi_{\phi}^{T}\left(x_{2}\right)\right.\right. \\
& \left.+x_{2} r \sqrt{\eta^{2}-1} \phi_{\phi}^{a}\left(x_{2}\right)\right] \cdot H_{1}\left(t_{1}\right)+\left[\left(r \eta-\frac{x_{1}}{2}\right) \phi_{\phi}^{v}\left(x_{2}\right)\right. \\
& \left.\left.+\left(r \sqrt{\eta^{2}-1}+\frac{x_{1}}{2}\right) \phi_{\phi}^{a}\left(x_{2}\right)\right] \cdot H_{2}\left(t_{2}\right)\right\},
\end{aligned}
$$$$
\begin{aligned}
A_{2}\left(q^{2}\right)= & \frac{(1+r)^{2}(\eta-r)}{2 r\left(\eta^{2}-1\right)} A_{1}\left(q^{2}\right) \\
& -8 \pi m_{B_{s}}^{2} C_{F} \frac{1+r}{\eta^{2}-r} \int d x_{1} d x_{2} \int b_{1} d b_{1} b_{2} d b_{2} \phi_{B_{s}}\left(x_{1}\right) \\
& \times\left\{\left[\left[\eta\left(1-x_{2} r^{2}\right)+r\left(x_{2}\left(2 \eta^{2}-1\right)-1\right)\right] \phi_{\phi}\left(x_{2}\right)\right.\right. \\
& +\left[1+2 x_{2} r^{2}-\left(1+2 x_{2}\right) r \eta\right] \phi_{\phi}^{t}\left(x_{2}\right) \\
& \left.+r\left(1-2 x_{2}\right) \sqrt{\eta^{2}-1} \phi_{\phi}^{s}\left(x_{2}\right)\right] \cdot H_{1}\left(t_{1}\right) \\
& +\left[\left[\left(r \eta-\frac{1}{2}\right) x_{1} \sqrt{\eta^{2}-1}-\left[r\left(r \eta-1-x_{1} \eta^{2}\right)+\frac{x_{1}(r+\eta)}{2}\right]\right] \phi_{\phi}\left(x_{2}\right)\right. \\
& \left.\left.+\left[x_{1}(r \eta-1)+\left(x_{2}-2\right) r \sqrt{\eta^{2}-1}\right] \phi_{\phi}^{s}\left(x_{2}\right)\right] \cdot H_{2}\left(t_{2}\right)\right\},
\end{aligned}
$$$$
T_{1}\left(q^{2}\right)=8 \pi m_{B_{s}}^{2} C_{F} \int d x_{1} d x_{2} \int b_{1} d b_{1} b_{2} d b_{2} \phi_{B_{s}}\left(x_{1}\right)
$$$$
\times\left\{\left[\left(1-2 x_{2}\right) r \phi_{\phi}^{v}\left(x_{2}\right)+\left(1+2 x_{2} r \eta-x_{2} r^{2}\right) \phi_{\phi}^{T}\left(x_{2}\right)\right.\right.
$$$$
\left.+\frac{1+2 x_{2} r^{2}-\left(1+2 x_{2}\right) r \eta}{\sqrt{\eta^{2}-1}} \phi_{\phi}^{a}\left(x_{2}\right)\right] \cdot H_{1}\left(t_{1}\right)
$$$$
+\left[\left[\left(1-\frac{x_{1}}{2}\right) r-\frac{x_{1}(r \eta-1)}{2 \sqrt{\eta^{2}-1}}\right] \phi_{\phi}^{v}\left(x_{2}\right)\right.
$$$$
\left.\left.+\left[\frac{r(\eta-r)}{\sqrt{\eta^{2}-1}}+\frac{x_{1}}{2}\left(r+\frac{r \eta-1}{\sqrt{\eta^{2}-1}}\right)\right] \phi_{\phi}^{a}\left(x_{2}\right)\right] \cdot H_{2}\left(t_{2}\right)\right\},
$$$$
T_{2}\left(q^{2}\right)=16 \pi m_{B_{s}}^{2} C_{F} \frac{r}{1-r^{2}} \int d x_{1} d x_{2} \int b_{1} d b_{1} b_{2} d b_{2} \phi_{B_{s}}\left(x_{1}\right)
$$$$
\times\left\{\left[\left(1-\left(1+2 x_{2}\right) r \eta+2 x_{2} r^{2}\right) \phi_{\phi}^{v}\left(x_{2}\right)+\left(x_{2} r \eta(2 \eta-r)-x_{2} r\right.\right.\right.
$$$$
\left.+\eta-r) \phi_{\phi}^{T}\left(x_{2}\right)+\left(1-2 x_{2}\right) r \sqrt{\eta^{2}-1} \phi_{\phi}^{a}\left(x_{2}\right)\right] \cdot H_{1}\left(t_{1}\right)
$$$$
+\left[\left[\frac{x_{2}}{2}\left(1+\frac{\eta}{\sqrt{\eta^{2}-1}}\right)(r \eta-1)+\left(r+\frac{x_{1}}{2 \sqrt{\eta^{2}-1}}\right)(\eta-r)\right] \phi_{\phi}^{v}\left(x_{2}\right)\right.
$$$$
\left.\left.+\left[\left(1-\frac{x_{1}}{2}\right) r \sqrt{\eta^{2}-1}+\frac{x_{1}}{2}(1-r \eta)\right] \phi_{\phi}^{a}\left(x_{2}\right)\right] \cdot H_{2}\left(t_{2}\right)\right\} \text {, }
$$ 


$$
\begin{aligned}
T_{3}\left(q^{2}\right)= & \frac{(1-r)^{2}(\eta+r)}{2 r\left(\eta^{2}-1\right)} T_{2}\left(q^{2}\right) \\
& -8 \pi m_{B_{s}}^{2} C_{F} \frac{1-r^{2}}{\eta^{2}-1} \int d x_{1} d x_{2} \int b_{1} d b_{1} b_{2} d b_{2} \phi_{B_{s}}\left(x_{1}\right) \\
& \times\left\{\left[\frac{\eta^{2}-\left(1+2 x_{2}\right) r \eta+2 x_{2} r^{2}}{\eta-r} \phi_{\phi}\left(x_{2}\right)+\left(1+x_{2} r \eta\right) \phi_{\phi}^{t}\left(x_{2}\right)\right.\right. \\
& \left.+x_{2} r \sqrt{\eta^{2}-1} \phi_{\phi}^{s}\left(x_{2}\right)\right] \cdot H_{1}\left(t_{1}\right) \\
& +\left[\left[r-\frac{x_{1}}{2}\left(\eta+\sqrt{\eta^{2}-1}\right)\right] \phi_{\phi}\left(x_{2}\right)\right. \\
& \left.\left.+\left(x_{1}+2 r \sqrt{\eta^{2}-1}\right) \phi_{\phi}^{s}\left(x_{2}\right)\right] \cdot H_{2}\left(t_{2}\right)\right\},
\end{aligned}
$$

where $r=m_{\phi} / m_{B_{s}}$, the twist-2 DAs $\left(\phi_{\phi}, \phi_{\phi}^{T}\right)$, and the twist-3 DAs $\left(\phi_{\phi}^{s, t}, \phi_{\phi}^{v, a}\right)$ have been defined in Equations (7) and (10). The function $H_{i}\left(t_{i}\right)$ in above equations is of the following form:

$H_{i}\left(t_{i}\right)=h_{i}\left(x_{1}, x_{2}, b_{1}, b_{2}\right) \cdot \alpha_{s}\left(t_{i}\right) \cdot S_{t}\left(x_{2}\right) \exp \left[-S_{a b}\left(t_{i}\right)\right], \quad$ for $i=(1,2)$.

The hard functions $h_{1,2}\left(x_{1}, x_{2}, b_{1}, b_{2}\right)$ come form the Fourier transform of virtual quark and gluon propagators and they can be defined by the following:

$$
\begin{aligned}
h_{1}= & K_{0}\left(\beta_{1} b_{1}\right)\left[\theta\left(b_{1}-b_{2}\right) I_{0}\left(\alpha_{1} b_{2}\right) K_{0}\left(\alpha_{1} b_{1}\right)\right. \\
& \left.+\theta\left(b_{2}-b_{1}\right) I_{0}\left(\alpha_{1} b_{1}\right) K_{0}\left(\alpha_{1} b_{2}\right)\right], \\
h_{2}= & K_{0}\left(\beta_{2} b_{1}\right)\left[\theta\left(b_{1}-b_{2}\right) I_{0}\left(\alpha_{2} b_{2}\right) K_{0}\left(\alpha_{2} b_{1}\right)\right. \\
& \left.+\theta\left(b_{2}-b_{1}\right) I_{0}\left(\alpha_{2} b_{1}\right) K_{0}\left(\alpha_{2} b_{2}\right)\right],
\end{aligned}
$$

where $K_{0}$ and $I_{0}$ are the modified Bessel functions and

$$
\begin{aligned}
& \alpha_{1}=m_{B_{s}} \sqrt{x_{2} r \eta^{+}}, \\
& \alpha_{2}=m_{B_{s}} \sqrt{x_{1} r \eta^{+}-r^{2}+r_{s}^{2}}, \\
& \beta_{1}=\beta_{2}=m_{B_{s}} \sqrt{x_{1} x_{2} r \eta^{+}},
\end{aligned}
$$

where $r=m_{\phi} / m_{B_{s}}$ and $r_{s}=m_{s} / m_{B_{s}}$. The hard scales $t_{i}$ in Equation (20) are chosen as the largest scale of the virtuality of the internal particles in the hard $b$-quark decay diagram, including $1 / b_{i}(i=1,2)$ :

$$
\begin{aligned}
& t_{1}=\max \left\{\alpha_{1}, \frac{1}{b_{1}}, \frac{1}{b_{2}}\right\}, \\
& t_{2}=\max \left\{\alpha_{2}, \frac{1}{b_{1}}, \frac{1}{b_{2}}\right\} .
\end{aligned}
$$

The threshold resummation factor $S_{t}(x)$ in Equation (20) is adopted from [49]:

$$
S_{t}=\frac{2^{1+2 c} \Gamma(3 / 2+c)}{\sqrt{\pi} \Gamma(1+c)}[x(1-x)]^{c},
$$

with a fitted parameter $c\left(Q^{2}\right)=0.04 Q^{2}-0.51 Q+1.87$ [39] and $Q^{2}=m_{B_{s}}^{2}\left(1-r^{2}\right)$ [69]. The function $S_{t}(x)$ is normalized to unity. The function $\exp \left[-S_{a b}(t)\right]$ in Equation (20) contains the Sudakov logarithmic corrections and the renormalization group evolution effects of both the wave functions and the hard scattering amplitude; for more details of function $\exp \left[-S_{a b}(t)\right]$, one can see References $[37,49]$.

\section{Observables for $B_{s} \longrightarrow \phi \ell^{+} \ell^{-}$Mode Decays}

In experimental analysis, the $\bar{B}_{s} \longrightarrow \phi \ell^{+} \ell^{-}$decay is treated as the four body differential decay distribution $\bar{B}_{s} \longrightarrow \phi(\longrightarrow$ $\left.K^{+} K^{-}\right) \ell^{+} \ell^{-}$and has been described in terms of the four kinematic variables $[10,11,13,19]$ : the lepton invariant mass squared $q^{2}$ and the three decay angles $\vec{\Omega}=\left(\cos \theta_{K}, \cos \theta_{\ell}\right.$, $\Phi)$. The angle $\theta_{K}$ is the angle between the direction of flight of $K^{+}$and $B_{s}$ meson in the rest frame of $\phi, \theta_{\ell}$ is the angle made by $\ell^{-}$with respect to the $B_{s}$ meson in the dilepton rest frame, and $\Phi$ is the azimuthal angle between the two planes formed by dilepton and $K^{+} K^{-}$.

With the hadronic and leptonic amplitudes defined in Equation (13), we write down the fourfold differential distribution of four-body $\bar{B}_{s} \longrightarrow \phi\left(\longrightarrow K^{+} K^{-}\right) \ell^{+} \ell^{-}$decay $[13,55$, 70, 71]:

$$
\frac{d^{4} \Gamma}{d q^{2} d \vec{\Omega}}=\frac{9}{32 \pi} I\left(q^{2}, \vec{\Omega}\right), \quad d \vec{\Omega}=d \cos \theta_{K} d \cos \theta_{\ell} d \Phi,
$$

where the functions $I\left(q^{2}, \vec{\Omega}\right)$ can be written in terms of a set of angular coefficients and trigonometric functions [70]:

$$
\begin{aligned}
I\left(q^{2}, \vec{\Omega}\right)= & \sum_{i} I_{i}\left(q^{2}\right) f_{i}(\vec{\Omega})=I_{1 s} \sin ^{2} \theta_{K}+I_{1 c} \cos ^{2} \theta_{K} \\
& +\left(I_{2 s} \sin ^{2} \theta_{K}+I_{2 c} \cos ^{2} \theta_{K}\right) \cos 2 \theta_{\ell} \\
& +I_{3} \sin ^{2} \theta_{K} \sin ^{2} \theta_{\ell} \cos 2 \Phi+I_{4} \sin 2 \theta_{K} \sin 2 \theta_{\ell} \cos \Phi \\
& +I_{5} \sin 2 \theta_{K} \sin \theta_{\ell} \cos \Phi+I_{6 s} \sin ^{2} \theta_{K} \cos \theta_{\ell} \\
& +I_{7} \sin 2 \theta_{K} \sin \theta_{\ell} \sin \Phi+I_{8} \sin 2 \theta_{K} \sin 2 \theta_{\ell} \sin \Phi \\
& +I_{9} \sin ^{2} \theta_{K} \sin ^{2} \theta_{\ell} \sin 2 \Phi .
\end{aligned}
$$

For the CP-conjugated mode $B_{s} \longrightarrow \phi\left(\longrightarrow K^{-} K^{+}\right) \ell^{+} \ell^{-}$, the corresponding expression of the angular decay distribution is as follows:

$$
\frac{d^{4} \bar{\Gamma}}{d q^{2} d \vec{\Omega}}=\frac{9}{32 \pi} \bar{I}\left(q^{2}, \vec{\Omega}\right)
$$

where the function $\bar{I}\left(q^{2}, \vec{\Omega}\right)$ is obtained from $I\left(q^{2}, \vec{\Omega}\right)$ in Equation (26) by making the complex conjugation for all weak phases in $I_{i}[70]$ and numerically by the following substitution:

$$
I_{1(c, s), 2(c, s), 3,4,7} \longrightarrow \bar{I}_{1(c, s), 2(c, s), 3,4,7}, I_{5,66,8,9} \longrightarrow-\bar{I}_{5,6 s, 8,9}
$$


TABLE 1: The explicit expressions of the angular coefficients $I_{i}\left(q^{2}\right)$ and $f_{i}(\vec{\Omega})$ appeared in Equation (26).

\begin{tabular}{lcc}
\hline$i$ & $I_{i}\left(q^{2}\right)$ & $f_{i}(\vec{\Omega})$ \\
\hline $1 s$ & $\left(\frac{3}{4}-\widehat{m}_{\ell}^{2}\right)\left[\left|\mathscr{A}_{\|}^{L}\right|^{2}+\left|\mathscr{A}_{\perp}^{L}\right|^{2}+\left|\mathscr{A}_{\|}^{R}\right|^{2}+\left|\mathscr{A}_{\perp}^{R}\right|^{2}\right]+4 \widehat{m}_{\ell}^{2} \operatorname{Re}\left[\mathscr{A}_{\perp}^{L} \mathscr{A}_{\perp}^{R *}+\mathscr{A}_{\|}^{L} \mathscr{A}_{\|}^{R *}\right]$ & $\sin ^{2} \theta_{K}$ \\
$1 c$ & $\left|\mathscr{A}_{0}^{L}\right|^{2}+\left|\mathscr{A}_{0}^{R}\right|^{2}+4 \widehat{m}_{\ell}^{2}\left[\left|\mathscr{A}_{t}\right|^{2}+2 \operatorname{Re}\left[\mathscr{A}_{0}^{L} \mathscr{A}_{0}^{R *}\right]\right]$ & $\cos ^{2} \theta_{K}$ \\
$2 s$ & $\frac{1}{4} \beta_{\ell}^{2}\left[\left|\mathscr{A}_{\|}^{L}\right|^{2}+\left|\mathscr{A}_{\perp}^{L}\right|^{2}+\left|\mathscr{A}_{\|}^{R}\right|^{2}+\left|\mathscr{A}_{\perp}^{R}\right|^{2}\right]$ & $\sin ^{2} \theta_{K} \cos 2 \theta_{\ell}$ \\
$2 c$ & $-\beta_{\ell}^{2}\left[\left|\mathscr{A}_{0}^{L}\right|^{2}+\left|\mathscr{A}_{0}^{R}\right|^{2}\right]$ & $\cos ^{2} \theta_{K} \cos 2 \theta_{\ell}$ \\
3 & 1 & $\sin ^{2} \theta_{K} \sin ^{2} \theta_{\ell} \cos 2 \Phi$ \\
4 & $\frac{1}{2} \beta_{\ell}^{2}\left[\left|\mathscr{A}_{\perp}^{L}\right|^{2}-\left|\mathscr{A}_{\|}^{L}\right|^{2}+\left|\mathscr{A}_{\perp}^{R}\right|^{2}-\left|\mathscr{A}_{\|}^{R}\right|^{2}\right]$ & $\sin 2 \theta_{K} \sin 2 \theta_{\ell} \cos \Phi$ \\
5 & $\sqrt{\frac{1}{2}} \beta_{\ell}^{2} \operatorname{Re}\left(\mathscr{A}_{0}^{L} \mathscr{A}_{\|}^{L *}+\mathscr{A}_{0}^{R} \mathscr{A}_{\|}^{R *}\right)$ & $\sin 2 \theta_{K} \sin \theta_{\ell} \cos \Phi$ \\
$6 s$ & $\sqrt{2} \beta_{\ell} \operatorname{Re}\left(\mathscr{A}_{0}^{L} \mathscr{A}_{\perp}^{L *}-\mathscr{A}_{0}^{R} \mathscr{A}_{\perp}^{R *}\right)$ & $\sin \theta_{K} \cos \theta_{\ell}$ \\
7 & $2 \beta_{\ell} \operatorname{Re}\left(\mathscr{A}_{\|}^{L} \mathscr{A}_{\perp}^{L *}-\mathscr{A}_{\|}^{R} \mathscr{A}_{\perp}^{R *}\right)$ & $\sin 2 \theta_{K} \sin \theta_{\ell} \sin \Phi$ \\
8 & $\sqrt{2} \beta_{\ell} \operatorname{Im}\left(\mathscr{A}_{0}^{L} \mathscr{A}_{\|}^{L *}-\mathscr{A}_{0}^{R} \mathscr{A}_{\|}^{R *}\right)$ & $\sin 2 \theta_{K} \sin 2 \theta_{\ell} \sin \Phi$ \\
9 & $\sqrt{\frac{1}{2}} \beta_{\ell}^{2} \operatorname{Im}\left(\mathscr{A}_{0}^{L} \mathscr{A}_{\perp}^{L *}+\mathscr{A}_{0}^{R} \mathscr{A}_{\perp}^{R *}\right)$ & $\sin \theta_{K} \sin { }^{2} \theta_{\ell} \sin 2 \Phi$ \\
\hline
\end{tabular}

The minus sign in Equation (28) is a result of the convention that, under the previous definitions of three angles $\left(\theta_{K}, \theta_{l}, \Phi\right)$, a CP transformation interchanges the lepton and antilepton, i.e., leading to the transformation $\theta_{\ell} \longrightarrow \theta_{\ell}$ $-\pi$ and $\Phi \longrightarrow-\Phi$.

The angular coefficients $I_{i}$, which are functions of $q^{2}$ only, are usually expressed in terms of the transverse amplitudes $[8,13]$. In the limit of massless leptons, there are six such complex amplitudes: $A_{0}^{L, R}, A_{\|}^{L, R}$, and $A_{\perp}^{L, R}$, where $L$ and $R$ refer to the chirality of the leptonic current. For the massive case, an additional complex amplitude $A_{t}$ is required, where the timelike component of the virtual gauge boson (which can later decay into dilepton) couples to an axial vector current.

In Table 1, we summarize the treatment of the angular distribution by decomposition of the angular coefficients $I_{i}$ $\left(q^{2}\right)$ into seven transverse amplitude $A_{\perp, \|, 0}^{L, R}$ and $A_{t}$ as well as the corresponding trigonometric factor $f_{i}(\vec{\Omega})$. Here, we will not consider scalar contribution to facilitate the comparison with Reference [35]. Notice that the distribution including lepton masses (but neglecting scalar $I_{6 c}=0$ ) contains eleven $I_{i}$ where only 10 of them are independent $[8,72]$. In the limit of massless leptons, it is easy to obtain the relations $I_{1 s}=3 I_{2 s}$ and $I_{1 c}=-I_{2 c}[70]$.

The seven transverse amplitudes $\mathscr{A}_{0}^{L, R}, \mathscr{A}_{\|}^{L, R}, \mathscr{A}_{\perp}^{L, R}$, and $\mathscr{A}_{t}$ of $B_{s} \longrightarrow \phi \ell^{+} \ell^{-}$decay, in turn, can be parameterized by means of the relevant form factors $[70,73]$ :

$$
\mathscr{A}_{\perp}^{L, R}=-N_{\ell} \sqrt{2 N_{\phi}} \sqrt{\lambda}\left[\left(C_{9}^{\text {eff }} \mp C_{10}\right) \frac{V\left(q^{2}\right)}{m_{B_{s}}+m_{\phi}}+2 \widehat{m}_{b} C_{7}^{\text {eff }} T_{1}\left(q^{2}\right)\right],
$$

$$
\begin{aligned}
\mathscr{A}_{\|}^{L, R}= & N_{\ell} \sqrt{2 N_{\phi}}\left[\left(C_{9}^{\text {eff }} \mp C_{10}\right)\left(m_{B_{s}}+m_{\phi}\right) \mathscr{A}_{1}\left(q^{2}\right)\right. \\
& \left.+2 \widehat{m}_{b} C_{7}^{\text {eff }}\left(m_{B_{s}}^{2}-m_{\phi}^{2}\right) T_{2}\left(q^{2}\right)\right], \\
\mathscr{A}_{0}^{L, R}= & \frac{N_{\ell} \sqrt{N_{\phi}}}{2 m_{\phi} \sqrt{q^{2}}}\left\{( C _ { 9 } ^ { \text { eff } } \mp C _ { 1 0 } ) \left[\left(m_{B_{s}}^{2}-m_{\phi}^{2}-q^{2}\right)\left(m_{B_{s}}+m_{\phi}\right) \mathscr{A}_{1}\left(q^{2}\right)\right.\right. \\
& \left.-\frac{\lambda}{m_{B_{s}}+m_{\phi}} \mathscr{A}_{2}\left(q^{2}\right)\right]+2 m_{b} C_{7}^{\text {eff }}\left[\left(m_{B_{s}}^{2}+3 m_{\phi}^{2}-q^{2}\right) T_{2}\left(q^{2}\right)\right. \\
& \left.\left.-\frac{\lambda}{m_{B_{s}}^{2}-m_{\phi}^{2}} T_{3}\left(q^{2}\right)\right]\right\}, \\
\mathscr{A}_{t}= & 2 N_{\ell} \sqrt{N_{\phi}} \frac{\sqrt{\lambda}}{\sqrt{q^{2}}} C_{10} \mathscr{A}_{0}\left(q^{2}\right),
\end{aligned}
$$

where $\lambda=\left(m_{B_{s}}^{2}-m_{\phi}^{2}-q^{2}\right)^{2}-4 m_{\phi}^{2} q^{2}, \widehat{m}_{b}=m_{b} / q^{2}$, and the normalization constants are given as follows:

$$
\begin{aligned}
& N_{\ell}=\frac{i \alpha_{e m} G_{F}}{4 \sqrt{2} \pi} V_{t b} V_{t s}^{*}, \\
& N_{\phi}=\frac{8 \sqrt{\lambda} q^{2}}{3 \times 256 \pi^{3} m_{B_{s}}^{3}} \sqrt{1-\frac{4 m_{\ell}^{2}}{q^{2}}} B\left(\phi \longrightarrow K^{+} K^{-}\right) .
\end{aligned}
$$

In numerical calculations, we take $B\left(\phi \longrightarrow K^{+} K^{-}\right)=$ 0.492 from PDG 2018 [74]. It is easy to see that the narrow width approximation works well in the case of $\phi$ meson since $\Gamma_{\phi} / m_{\phi}=4.17 \times 10^{-3} \sim 0$.

Analogous to Reference [70], to separate $\mathrm{CP}$-conserving and $\mathrm{CP}$-violating effects, one can define the $\mathrm{CP}$-averaged 
angular coefficients $S_{i}$ and CP asymmetry angular coefficients $A_{i}$ normalized by the differential (CP-averaged) decay rate to reduce the theoretical uncertainties:

$$
\begin{aligned}
& S_{i}=\frac{I_{i}+\bar{I}_{i}}{d(\Gamma+\bar{\Gamma}) / d q^{2}}, \\
& A_{i}=\frac{I_{i}-\bar{I}_{i}}{d(\Gamma+\bar{\Gamma}) / d q^{2}},
\end{aligned}
$$

where $I_{i}$ and $\bar{I}_{i}$ have been defined in Equations (26), (27), and (28) and Table 1, and the differential decay rate reads (analogously for $\bar{\Gamma}$ )

$$
\frac{d \Gamma}{d q^{2}}=\frac{1}{4}\left(3 I_{1 c}+6 I_{1 s}-I_{2 c}-2 I_{2 s}\right)
$$

Based on the definition of $S_{i}$, one can find the relation $3 S_{1 c}+6 S_{1 s}-S_{2 c}-2 S_{2 s}=4$. Consequently, all established observables can be expressed in terms of $S_{i}$ and $A_{i}$ :

(1) The CP asymmetry:

$A_{\mathrm{CP}}\left(q^{2}\right)=\frac{d \Gamma / d q^{2}-d \bar{\Gamma} / d q^{2}}{d \Gamma / d q^{2}+d \bar{\Gamma} / d q^{2}}=\frac{1}{4}\left(3 A_{1 c}+6 A_{1 s}-A_{2 c}-2 A_{2 s}\right)$.

(2) The lepton forward-backward (CP) asymmetry:

$$
\begin{aligned}
& A_{\mathrm{FB}}\left(q^{2}\right)=\frac{\left[\int_{0}^{1}-\int_{-1}^{0}\right] d \cos \theta_{\ell}\left(d^{2}(\Gamma-\bar{\Gamma}) / d q^{2} d \cos \theta_{\ell}\right)}{d(\Gamma+\bar{\Gamma}) / d q^{2}}=\frac{3}{4} S_{6 s}, \\
& A_{\mathrm{FB}}^{\mathrm{CP}}\left(q^{2}\right)=\frac{\left[\int_{0}^{1}-\int_{-1}^{0}\right] d \cos \theta_{\ell}\left(d^{2}(\Gamma+\bar{\Gamma}) / d q^{2} d \cos \theta_{\ell}\right)}{d(\Gamma+\bar{\Gamma}) / d q^{2}}=\frac{3}{4} A_{6 s} .
\end{aligned}
$$

(3) The $\phi$ polarization fractions:

$$
\begin{aligned}
& F_{L}\left(q^{2}\right)=\frac{1}{4}\left(3 S_{1 c}-S_{2 c}\right), \\
& F_{T}\left(q^{2}\right)=\frac{1}{2}\left(3 S_{1 s}-S_{2 s}\right) .
\end{aligned}
$$

In the massless limit, since the $\mathrm{CP}$-averaged observable $S_{1(c, s), 2(c, s)}$ obey the relations $S_{1 s}=3 S_{2 s}$ and $S_{1 c}=-S_{2 c}$, the definitions of the polarization fractions can be simplified directly as follows:

$$
\begin{gathered}
F_{L}\left(q^{2}\right)=S_{1 c}=-S_{2 c} \\
F_{T}\left(q^{2}\right)=\frac{4}{3} S_{1 s}=4 S_{2 s} .
\end{gathered}
$$

(4) The clean (no S-wave pollution) observables $P_{1,2,3}$ and $P_{4,5,6}$ in the natural basis can be defined in terms of the coefficients $S_{i}$ through the following relations $[71,75,76]$ :

$$
\begin{aligned}
P_{1} & =\frac{S_{3}}{2 S_{2 s}}, \\
P_{2} & =\beta_{\ell} \frac{S_{6 s}}{8 S_{2 s}}, \\
P_{3} & =-\frac{S_{9}}{4 S_{2 s}}, \\
P_{4}^{\prime} & =\frac{S_{4}}{\sqrt{S_{1 c} S_{2 s}}}, \\
P^{\prime} & =\frac{\beta_{\ell} S_{5}}{2 \sqrt{S_{1 c} S_{2 s}}}, \\
P_{6}^{\prime} & =-\frac{\beta_{\ell} S_{7}}{2 \sqrt{S_{1 c} S_{2 s}}}, \\
P^{\prime}{ }_{8} & =-\frac{S_{8}}{\sqrt{S_{1 c} S_{2 s}}},
\end{aligned}
$$

where $\beta_{\ell}=\sqrt{1-4 m_{\ell}^{2} / q^{2}}$.

(5) In the massless limit of leptons, the optimized observables $P_{i}^{()}$[8] can be transformed as the following form:

$$
\begin{aligned}
P_{1} & =\frac{2 S_{3}}{F_{T}}, \\
P_{2} & =\frac{S_{6 s}}{2 F_{T}}, \\
P_{3} & =\frac{-S_{9}}{F_{T}}, \\
P_{4}^{\prime} & =\frac{2 S_{4}}{\sqrt{F_{L}\left(1-F_{L}\right)}}, \\
P_{5}^{\prime} & =\frac{S_{5}}{\sqrt{F_{L}\left(1-F_{L}\right)}}, \\
P_{6}^{\prime} & =-\frac{S_{7}}{\sqrt{F_{L}\left(1-F_{L}\right)}}, \\
P_{8}^{\prime} & =-\frac{2 S_{8}}{\sqrt{F_{L}\left(1-F_{L}\right)}} .
\end{aligned}
$$


One should know that our definitions of the CPaveraged angular coefficients $S_{i}$, the CP asymmetry angular coefficients $A_{i}$, and the clean observable $P_{1,2,3}$ and $P_{4,5,6}$ differ from those adopted by the LHCb Collaboration. To be specific, the reasons are the following:

(1) Our conventions for the angles to define the $B_{s}$ $\longrightarrow \phi \ell^{+} \ell^{-}$kinematics are identical to the Reference [70] but different from the LHCb choices [13, 31]. The corresponding relations are the following:

$$
\begin{aligned}
\theta_{K}^{\mathrm{LHCb}} & =\theta_{K}, \\
\theta_{\ell}^{\mathrm{LHCb}} & =\pi-\theta_{\ell}, \\
\Phi^{\mathrm{LHCb}} & =-\Phi .
\end{aligned}
$$

Some angular coefficients $I_{i}, S_{i}$, and $A_{i}$, consequently, will have different signs:

$$
\begin{gathered}
I_{4,6,7,9}^{\mathrm{LHCb}}=-I_{4,6 s, 7,9}, \\
S_{4,6,7,9}^{\mathrm{LHCb}}=-S_{4,6 s, 7,9}, \\
A_{4,6,7,9}^{\mathrm{LHCb}}=-A_{4,6 s, 7,9} .
\end{gathered}
$$

Other remaining coefficients $I_{i}\left(S_{i}\right.$ and $\left.A_{i}\right)$, however, have the same sign in both conventions.

(2) Our definitions of the clean observables $P_{1,2,3}$ and $P_{4,5,6,8}$ in Equation (38) in terms of $S_{i}$ may be different from those defined and used by the LHCb Collaboration, for example, in Reference [13]. The resultant differences of the sign and normalization are of the following:

$$
\begin{aligned}
P_{1}^{\mathrm{LHCb}} & =P_{1}, \\
P_{2,3}^{\mathrm{LHCb}} & =-P_{2,3}, \\
P_{4,8}^{\prime}{ }^{\mathrm{LHCb}} & =-\frac{1}{2} P_{4,8}^{\prime}, \\
P_{5,6}^{\prime}{ }^{\mathrm{LHCb}} & =P_{5,6}^{\prime} .
\end{aligned}
$$

For more details about the angular conventions of the angular observables of the semileptonic decays $B_{(s)} \longrightarrow$ $\mathrm{Vl}^{+} \mathrm{l}^{-}$, one can see Reference [77].

\section{Numerical Results and Discussions}

In the numerical calculations, we use the following input parameters (here, masses and decay constants are in units of $\mathrm{GeV})[42,74]$ :

$$
\begin{aligned}
\Lambda_{\overline{\mathrm{MS}}}^{f=4} & =0.250, \\
\tau_{B_{s}^{0}} & =1.509 \mathrm{ps}, \\
m_{b} & =4.8, \\
m_{W} & =80.38, \\
m_{\phi} & =1.019, \\
m_{B_{s}} & =5.367, \\
m_{e} & =0.000511, \\
m_{\mu} & =0.105, \\
m_{\tau} & =1.777, \\
f_{B_{s}} & =0.23, \\
f_{\phi} & =0.231(4), \\
f_{\phi}^{\perp} & =0.20(1), \\
a_{2 \phi}^{\|} & =0.18(8), \\
a_{2 \phi}^{\perp} & =0.14(7) .
\end{aligned}
$$

For the CKM matrix elements and angles, we adopt the following values as given in Reference [74]:

$$
\begin{aligned}
V_{t b} & =1.019(25), \\
V_{u s} & =0.2243(5), \\
\left|V_{t s}\right| & =(39.4 \pm 2.3) \times 10^{-3}, \\
\left|V_{u b}\right| & =(3.94 \pm 0.36) \times 10^{-3}, \\
2 \beta_{s} & =0.021(31), \\
\gamma & =\left(73.5_{-5.1}^{+4.2}\right)^{\circ} .
\end{aligned}
$$

4.1. The Form Factors. For the considered semileptonic decays, the differential decay rates and other physical observables strongly rely on the value and the shape of the relevant form factors $V\left(q^{2}\right), A_{0,1,2}\left(q^{2}\right)$ and $T_{1,2,3}\left(q^{2}\right)$ for $B_{s}$ $\longrightarrow \phi \ell^{+} \ell^{-}$decays. These form factors have been calculated in rather different theories or models [23, 36-38, 42]. Since the PQCD predictions for the considered form factors are valid only at the large hadronic recoil (low- $\left.q^{2}\right)$ region, we usually calculate explicitly the values of the relevant form factors at the low- $q^{2}$ region, say $0 \leq q^{2} \leq m_{\tau}^{2}$, and then make an extrapolation for all relevant form factors from the low $-q^{2}$ towards the high $-q^{2}$ region by using the pole model parametrization $[78,79]$ or other different methods.

In References [80-82], we developed a new method: the so-called "PQCD+Lattice" approach. Here, we still use the PQCD approach to evaluate the form factors at the low $-q^{2}$ region, but take those currently available lattice QCD results for the relevant form factors at the high- $q^{2}$ region as the lattice QCD input to improve the extrapolation of the form factors up to $q_{\max }^{2}$. In References [81, 82], we used the Bourrely-Caprini-Lellouch (BCL) parametrization 
TABLE 2: The PQCD predictions of the seven form factors $F_{B_{s}}^{i} \longrightarrow \phi(0)$ with the theoretical uncertainties from the variations of the parameters $\omega_{B_{s}}, a_{2 \phi}^{\|, \perp}, f_{\phi}$, and $f_{\phi}^{T}$.

\begin{tabular}{lc}
\hline$F_{B_{s} \longrightarrow \phi}^{i}(0)$ & PQCD predictions \\
\hline$V(0)$ & $0.311_{-0.051}^{+0.063}\left(\omega_{B_{s}}\right)_{-0.007}^{+0.008}\left(a_{2 \phi}^{\perp}\right) \pm 0.004\left(f_{\phi}\right) \pm 0.004\left(f_{\phi}^{T}\right)$ \\
$A_{0}(0)$ & $0.262_{-0.038}^{+0.046}\left(\omega_{B_{s}}\right)_{-0.008}^{+0.009}\left(a_{2 \phi}^{\|}\right) \pm 0.002\left(f_{\phi}\right) \pm 0.008\left(f_{\phi}^{T}\right)$ \\
$A_{1}(0)$ & $0.247_{-0.041}^{+0.051}\left(\omega_{B_{s}}\right)_{-0.006}^{+0.005}\left(a_{2 \phi}^{\perp}\right) \pm 0.003\left(f_{\phi}\right) \pm 0.003\left(f_{\phi}^{T}\right)$ \\
$A_{2}(0)$ & $0.239_{-0.042}^{+0.054}\left(\omega_{B_{s}}\right) \pm 0.009\left(a_{2 \phi}^{\|, \perp}\right) \pm 0.004\left(f_{\phi}\right) \pm 0.001\left(f_{\phi}^{T}\right)$ \\
$T_{1,2}(0)$ & $0.264_{-0.042}^{+0.052}\left(\omega_{B_{s}}\right) \pm 0.006\left(a_{2 \phi}^{\perp}\right) \pm 0.003\left(f_{\phi}\right) \pm 0.005\left(f_{\phi}^{T}\right)$ \\
$T_{3}(0)$ & $0.196_{-0.033}^{+0.041}\left(\omega_{B_{s}}\right) \pm 0.008\left(a_{2 \phi}^{\|, \perp}\right) \pm 0.003\left(f_{\phi}\right) \pm 0.001\left(f_{\phi}^{T}\right)$ \\
\hline
\end{tabular}

TABLE 3: The central values of the theoretical predictions for the form factors of $B_{s} \longrightarrow \phi$ transitions at $q^{2}=0$ from different theories or models [17, 20, 21, 23, 42, 85-91].

\begin{tabular}{|c|c|c|c|c|c|c|}
\hline This work & $\begin{array}{l}V(0) \\
0.311\end{array}$ & $\begin{array}{c}A_{0}(0) \\
0.262\end{array}$ & $\begin{array}{l}A_{1}(0) \\
0.247\end{array}$ & $\begin{array}{l}A_{2}(0) \\
0.239\end{array}$ & $\begin{array}{c}T_{1,2}(0) \\
0.264\end{array}$ & $\begin{array}{l}T_{3}(0) \\
0.196\end{array}$ \\
\hline PQCD [42] & 0.25 & 0.30 & 0.19 & - & - & \\
\hline PQCD [85] & 0.26 & 0.31 & 0.18 & 0.12 & 0.23 & 0.19 \\
\hline LCSR [23] & 0.387 & 0.389 & 0.296 & - & 0.309 & - \\
\hline LCSR [20] & 0.434 & 0.474 & 0.311 & 0.234 & 0.349 & 0.175 \\
\hline LCSR [86] & 0.433 & 0.382 & 0.296 & 0.255 & 0.348 & 0.254 \\
\hline QCDSR [87] & 0.45 & 0.30 & 0.32 & 0.30 & 0.33 & 0.22 \\
\hline RDA [88] & 0.44 & 0.42 & 0.34 & 0.31 & 0.38 & 0.26 \\
\hline RQM [89] & 0.406 & 0.322 & 0.320 & 0.318 & 0.275 & 0.133 \\
\hline SCET [90] & 0.329 & 0.279 & 0.232 & 0.210 & 0.276 & 0.170 \\
\hline HQEFT [21] & 0.339 & 0.269 & 0.271 & 0.212 & 0.299 & 0.191 \\
\hline SQEH [91] & 0.259 & 0.311 & 0.194 & - & - & - \\
\hline CQM [17] & 0.31 & 0.28 & 0.27 & 0.27 & 0.27 & 0.18 \\
\hline
\end{tabular}

method $[83,84]$ instead of the traditional pole model parametrization.

In Table 2, we list the PQCD predictions for all seven relevant form factors $V(0), A_{0,1,2}(0)$, and $T_{1,2,3}(0)$ for $B_{s}$ $\longrightarrow \phi$ transition at the point $q^{2}=0$. The dominant theoretical errors come from the uncertainties of the parameter $\omega_{B_{s}}=0.50 \pm 0.05$ [42], the Gegenbauer moments $a_{2 \phi}^{\|}=$ $0.18 \pm 0.08$ and $a_{2 \phi}^{\perp}=0.14 \pm 0.07$ [42, 46, 47, 51], and the decay constants $f_{\phi}=0.231 \pm 0.004 \mathrm{GeV}$ and $f_{\phi}^{T}=0.20 \pm$ $0.01 \mathrm{GeV}$ [42].

In Table 3, as a comparison, we also list the central values of the theoretical predictions for the form factors $F_{B_{s} \longrightarrow \phi}^{i}(0)$ at $q^{2}=0$ evaluated in the PQCD approach [42, $85]$ and in other different theories or models [17, 20, 21, $23,86-91]$. One can see that there exist always some differences between different authors, even among the authors using the same approach. Taking the calculations based on the LCSR method as an example, the authors of Reference [23] introduced the hadronic input parameters, Ball and Zwicky considered the one-loop radiative corrections [20], and Yilmaz included the radiative and higher twist corrections and $\mathrm{SU}(3)$ breaking effects [86].

In Table 4 , for the $B_{s} \longrightarrow \phi$ transition form factors $(V$, $\left.A_{0,1,2}, T_{1,2,3}\right)$, we quote directly the values of the lattice QCD results at two or three reference points of the high- $q^{2}$ region, say $q^{2}=12,16 \mathrm{GeV}^{2}$ and $q_{\max }^{2}=\left(m_{B_{s}}-m_{\phi}\right)^{2} \approx 18.9$ $\mathrm{GeV}^{2}$, as listed in Table XXXI of Reference [92]. In Reference [92], the authors defined the helicity form factors $A_{12}$ $\left(q^{2}\right)$ and $T_{23}\left(q^{2}\right)$ from the ordinary form factors $A_{1,2}\left(q^{2}\right)$ and $T_{2,3}\left(q^{2}\right)$ :

$$
\begin{aligned}
& A_{12}\left(q^{2}\right)=\frac{\left(m_{B_{s}}+m_{\phi}\right)^{2}\left(m_{B_{s}}^{2}-m_{\phi}^{2}-q^{2}\right) A_{1}\left(q^{2}\right)-\lambda\left(q^{2}\right) A_{2}\left(q^{2}\right)}{16 m_{B_{s}} m_{\phi}^{2}\left(m_{B_{s}}+m_{\phi}\right)}, \\
& T_{23}\left(q^{2}\right)=\frac{m_{B_{s}}+m_{\phi}}{8 m_{B_{s}} m_{\phi}^{2}}\left[\left(m_{B_{s}}^{2}+3 m_{\phi}^{2}-q^{2}\right) T_{2}\left(q^{2}\right)-\frac{\lambda\left(q^{2}\right) T_{3}\left(q^{2}\right)}{m_{B_{s}}^{2}-m_{\phi}^{2}}\right],
\end{aligned}
$$


TABLE 4: The values for the lattice QCD results of the relevant $B_{s} \longrightarrow \phi$ transition form factors at two or three reference points [92].

\begin{tabular}{lccccccc}
\hline$q^{2}$ & $V\left(q^{2}\right)$ & $A_{0}\left(q^{2}\right)$ & $A_{1}\left(q^{2}\right)$ & $A_{2}\left(q^{2}\right)$ & $T_{1}\left(q^{2}\right)$ & $T_{2}\left(q^{2}\right)$ & $T_{3}\left(q^{2}\right)$ \\
\hline 12 & $0.77(6)$ & $0.90(6)$ & $0.44(3)$ & $0.48(4)$ & $0.69(4)$ & $0.45(3)$ & $0.46(4)$ \\
16 & $1.19(7)$ & $1.32(7)$ & $0.52(3)$ & $0.54(4)$ & $0.99(5)$ & $0.53(3)$ & $0.70(5)$ \\
18.9 & $1.74(10)$ & $1.85(10)$ & $0.62(3)$ & - & $1.36(8)$ & $0.62(3)$ & - \\
\hline
\end{tabular}

where the kinematic variable $\lambda\left(q^{2}\right)=\left(t_{+}-q^{2}\right)\left(t_{-}-q^{2}\right)$ with $t_{ \pm}=\left(m_{B_{s}} \pm m_{\phi}\right)^{2}$. From above two equations and the numerical values of $\left(A_{1}\left(q^{2}\right), T_{2}\left(q^{2}\right), A_{12}\left(q^{2}\right), T_{23}\left(q^{2}\right)\right)$ as given in Reference [92], we can find the corresponding lattice QCD results of $A_{2}\left(q^{2}\right)$ and $T_{3}\left(q^{2}\right)$ at the two points $q^{2}=(12,16)$ $\mathrm{GeV}^{2}$ by direct numerical calculations. When $q^{2} \longrightarrow q_{\max }^{2}$ $=\left(m_{B_{s}}-m_{\phi}\right)^{2}$, however, the parameter $\lambda\left(q_{\max }^{2}\right)$ in Equation (45) is also approaching zero simultaneously; one therefore cannot determine $A_{2}\left(q_{\max }^{2}\right)$ and $T_{3}\left(q_{\max }^{2}\right)$ reliably from the values of $A_{12}\left(q_{\max }^{2}\right)$ and $T_{23}\left(q_{\max }^{2}\right)$ as given in Reference [92]. Consequently, $A_{2}\left(q_{\max }^{2}\right)$ and $T_{3}\left(q_{\max }^{2}\right)$ are absent in Table 4.

In this work, we will use both the PQCD factorization approach and the "PQCD+Lattice" approach to evaluate all relevant form factors over the whole range of $q^{2}$.

(1) In the PQCD approach, we use the definitions and formulae to calculate the values of all relevant form factors $V\left(q^{2}\right), A_{0,1,2}\left(q^{2}\right)$, and $T_{1,2,3}\left(q^{2}\right)$ at some points in the region of $0 \leq q^{2} \leq m_{\tau}^{2}$. We then make the extrapolation for these form factors to the large $q^{2}$ region up to $q_{\max }^{2}$ by using the selected parametrization method directly

(2) In the "PQCD+Lattice" approach, we take the lattice QCD results of the form factors at the points of $q^{2}$ $=(12,16,18.9) \mathrm{GeV}^{2}$ as the new input in the high$q^{2}$ region and then make a combined fit of the PQCD results in low $-q^{2}$ region and the lattice QCD results in high- $q^{2}$ region to determine the relevant parameters $b_{k}^{i}$ in the $z$-series expansion and then complete the extrapolation

(3) For both approaches, we always use the modelindependent $z$-series parametrization, which is based on a rapidly converging series in the parameter $z$, as in References $[23,55]$ to make the extrapolation. The entire cut $q^{2}$-plane will be mapped onto the unit disc $\left|z\left(q^{2}\right)\right| \leq 1$ under the conformal transformation as [93]

$$
z\left(q^{2}\right)=\frac{\sqrt{t_{+}-q^{2}}-\sqrt{t_{+}-t_{0}}}{\sqrt{t_{+}-q^{2}}+\sqrt{t_{+}-t_{0}}}
$$

where $t_{ \pm}=\left(m_{B_{s}} \pm m_{\phi}\right)^{2}$ and $0 \leq t_{0}<t_{-}$is an auxiliary parameter which can be optimized to reduce the maximum value of $\left|z\left(q^{2}\right)\right|$ in the physical range of the form factors and will be taken in the same way as in Reference [94]: $t_{0}=t_{+}(1-$ $\left.\sqrt{1-t_{-} / t_{+}}\right)$. The form factors are finally parameterized in the BCL version of the $z$-series expansion [83].

$$
\begin{aligned}
F_{B_{s} \longrightarrow \phi}^{i}\left(q^{2}\right) & =\frac{F_{B_{s} \longrightarrow \phi}^{i}(0)}{1-q^{2} / m_{i, \text { pole }}^{2}}\left\{1+\sum_{k=1}^{N} b_{k}^{i}\left[z\left(q^{2}, t_{0}\right)^{k}-z\left(0, t_{0}\right)^{k}\right]\right\} \\
& =\frac{F_{B_{s} \longrightarrow \phi}^{i} \longrightarrow(0)}{1-q^{2} / m_{i, \text { pole }}^{2}}\left\{1+b_{1}^{i}\left[z\left(q^{2}, t_{0}\right)-z\left(0, t_{0}\right)\right]\right\}+\cdots
\end{aligned}
$$

Since the term $\left|z\left(q^{2}, t_{0}\right)\right|^{2} \leq 0.04$ in the whole considered $q^{2}$ region, the high-order $N \geq 2$ terms in Equation (48) should be very small in magnitude and therefore can be neglected. After the truncation at $N=1$, the coefficient $b_{1}^{i}$ for the corresponding form factor $F_{B_{s} \longrightarrow \phi}^{i}\left(q^{2}\right)$ can be determined by fitting to the PQCD predictions at low $-q^{2}$ region and the lattice QCD results in the high $-q^{2}$ region. Taking the form factor $A_{2}\left(q^{2}\right)$ as an example, we calculate $A_{2}\left(q^{2}\right)$ first by employing the PQCD approach in the sixteen points in the low $0 \leq q^{2} \leq m_{\tau}^{2}$ region, take the lattice $\mathrm{QCD}$ results $A_{2}(12)=0.48 \pm 0.04$ and $A_{2}(16)=0.54 \pm 0.04$ as additional input, and finally make the fitting for the parameter $b_{1}^{A_{2}}$ and find that

$$
b_{1}^{A_{2}}=-1.820 \pm 0.148(\text { standard error })
$$

with the goodness-of-fit $R^{2}=0.9978$. For other form factors $F^{i}\left(q^{2}\right)$, we find the results by following the same kind of procedure. The input values of the various $\bar{s} b$-resonance mass $m_{i \text {,pole }}$ in Equation (48) can be found from Reference [74] and are collected in Table 5. For further discussions on the systematic uncertainties due to the dependence of truncation schemes and on the implementation of the strong unitary constraints, one can see References [94, 95].

(4) In Figure 1, we show the theoretical predictions of the form factors $V\left(q^{2}\right), A_{0,1,2}\left(q^{2}\right)$, and $T_{1,2,3}\left(q^{2}\right)$ for $B_{s} \longrightarrow \phi$ transition based on the PQCD approach (red curves) and the "PQCD+Lattice" approach (blue curves) with the extrapolation from $q^{2}=0$ to $q_{\max }^{2}=\left(m_{B_{s}}-m_{\phi}\right)^{2}$ by applying the $z$-series parameterizations. The shaded bands represent the total theoretical error obtained by adding in quadrature of the separate errors from the uncertainty of the parameter $\omega_{B_{s}}, a_{2 \phi}^{\|, \perp}, f_{\phi}$, and $f_{\phi}^{\perp}$. The black error bars in the low $-q^{2}$ region correspond to the PQCD 
TABLE 5: The masses $m_{i \text {,pole }}$ in Equation (48) for the form factors $F_{B_{s} \longrightarrow \phi}^{i}\left(q^{2}\right)[23]$.

\begin{tabular}{lcc}
\hline$F_{B_{s} \longrightarrow \phi}^{i}\left(q^{2}\right)$ & $\bar{s} b\left(J^{P}\right)$ & $m_{i, \text { pole }}(\mathrm{GeV})$ \\
$A_{0}\left(q^{2}\right)$ & $B_{s}\left(0^{-}\right)$ & 5.366 \\
$V\left(q^{2}\right), T_{1}\left(q^{2}\right)$ & $B_{s}^{*}\left(1^{-}\right)$ & 5.415 \\
$A_{1,2}\left(q^{2}\right), T_{2,3}\left(q^{2}\right)$ & $B_{s 1}\left(1^{+}\right)$ & 5.829 \\
\hline
\end{tabular}

predictions of the corresponding form factors, while the error bars in the high- $q^{2}$ region are the currently known lattice QCD results as collected in Table 3

4.2. Numerical Results. We now proceed to explore the phenomenological aspects of the cascade decays $B_{s} \longrightarrow \phi(\longrightarrow$ $\left.K^{-} K^{+}\right) \ell^{+} \ell^{-}$, which allow us to define and compute a number of physical observables and compare them with those measured by experiments. We first compare our results for the branching ratios and angular observables with the experimental data reported by the LHCb Collaboration [31, 33]. As studied systematically in the last section, the physical observables accessible in the semileptonic decays $B_{s} \longrightarrow \phi$ $\ell^{+} \ell^{-}$are the CP-averaged differential branching fraction $d$ $B / d q^{2}[31,33]$, the $\mathrm{CP}$-averaged $\phi$ meson longitudinal polarization fraction $F_{L}$, the forward-backward asymmetry $A_{\mathrm{FB}}$, the angular coefficients $S_{i}$ and $A_{i}$, and the optimized observables $P_{i}$ and $P_{j}^{\prime}$ [25]. The CP asymmetry angular coefficients $A_{5,6,8,9}$ in the SM are induced by the weak phase from the CKM matrix. For the $b \longrightarrow s$ transition, the CP asymmetries proportional to $\operatorname{Im}\left(V_{u b} V_{u s}^{*} / V_{t b} V_{t s}^{*}\right)$, which is of order $10^{-2}$ [19] as measured by the LHCb Collaboration (see Table 3 in Reference [31]), but the statistical uncertainties are still large. For these reasons, we will focus on the CPaveraged quantities when taking the binned observables into consideration.

We begin with the branching ratios of the decays $B_{s}$ $\longrightarrow \phi \ell^{+} \ell^{-}$. From the differential decay rates as defined in Equation (32), it is straightforward to make the integration over the range of $4 m_{\ell}^{2} \leq q^{2} \leq\left(m_{B_{s}}-m_{\phi}\right)^{2}$. In order to be consistent with the choices made by LHCb Collaboration in their data analysis, we here also cut off the regions of dilepton-mass squared around the charmonium resonances $J / \psi(1 S)$ and $\psi(2 S)$; i.e., $8.0<q^{2}<11.0 \mathrm{GeV}^{2}$ and $12.5<q^{2}$ $<15.0 \mathrm{GeV}^{2}$ for $\ell=(e, \mu, \tau)$ cases. We display the PQCD and "PQCD+Lattice" predictions for the differential branching ratios $d B / d q^{2}$ in Figure 2 for the cases of $l=(\mu, \tau)$, including currently available $\mathrm{LHCb}$ results in six or eight bins of $q^{2}[31,33]$ indicated by the crosses for $B_{s} \longrightarrow \phi \mu^{+}$ $\mu^{-}$decay. From Figure 2, one can see that both PQCD and "PQCD+Lattice" predictions for the differential branching ratios do agree well with the $\mathrm{LHCb}$ results within the still large errors. Since the theoretical prediction for the differential branching ratio of the electron mode is almost identical with the one of the muon, we do not draw the figure of $d B$ $\left(B_{s} \longrightarrow \phi e^{+} e^{-}\right) / d q^{2}$ in Figure 2 .
In Table 6, we present the theoretical predictions of the total branching fractions for $B_{s} \longrightarrow \phi \ell^{+} \ell^{-}$with $\ell=(e, \mu, \tau)$ obtained by the integration over the six $q^{2}$ bins using the PQCD (the first row) and "PQCD+Lattice" approach (the second row), respectively. The major theoretical errors from different sources, such as the form factors (FFs) as listed in Table 2, the scale $\mu$, and the CKM matrix element $V_{t b}$ and $V_{t s}$, are also listed. As in Reference [31], a correction factor $f_{\text {veto }}=1.52$ is applied to account for the contribution in the veto $q^{2}$ bins for $\ell=(e, \mu)$ cases. As a comparison, we also show the $\mathrm{LHCb}$ measured value $B\left(B_{s} \longrightarrow \phi \mu^{+} \mu^{-}\right)=(7.9$ $\left.7_{-0.80}^{+0.81}\right) \times 10^{-7} \quad[31]$ and $\left(8.14_{-0.47}^{+0.47}\right) \times 10^{-7}$ [33] and the QCDSR predictions $B\left(B_{s} \longrightarrow \phi \ell^{+} \ell^{-}\right)$for all three decay modes [87]. For $B_{s} \longrightarrow \phi \mu^{+} \mu^{-}$decay, for instance, the theoretical predictions and the $\mathrm{LHCb}$ measurement $[31,33$ ] (in unit of $10^{-7}$ ) are the following:

$$
B\left(B_{s} \longrightarrow \phi \mu^{+} \mu^{-}\right)= \begin{cases}7.07_{-2.34}^{+3.43}, & \text { in PQCD, } \\ 6.76_{-1.25}^{+1.52}, & \text { in PQCD + Lattice, } \\ 7.06_{-1.59}^{+1.59}, & \text { in QCDSR [87], } \\ 7.97_{-0.80}^{+0.81}, & \text { LHCb [31], } \\ 8.14_{-0.47}^{+0.47}, & \text { LHCb [33]. }\end{cases}
$$

From the numerical results in above equation and Table 6, one can see that

(1) The PQCD and "PQCD+Lattice" predictions for the branching ratio $B\left(B_{s} \longrightarrow \phi \ell^{+} \ell^{-}\right)$with $\ell=(e, \mu, \tau)$ do agree well with each other within the errors, while the "PQCD+ Lattice" predictions of $B\left(B_{s} \longrightarrow \phi \ell^{+} \ell^{-}\right)$ have smaller errors than those of the PQCD predictions

(2) Both PQCD and "PQCD+Lattice" predictions of $B($ $\left.B_{s} \longrightarrow \phi \mu^{+} \mu^{-}\right)$do agree well with currently available LHCb measured values [31, 33] within errors. For the electron and tau mode, however, we have to wait for the future experimental measurements

(3) For all three decay modes, our theoretical predictions of the branching ratios do agree well with the theoretical predictions obtained from the QCD sum rule [87]

Since the large theoretical uncertainties of the branching ratios could be largely canceled in the ratio of the branching ratios of $B_{s} \longrightarrow \phi \ell^{+} \ell^{-}$decays, one can define and check the physical observables $R_{\phi}^{e \mu}$ and $R_{\phi}^{\mu \tau}$ [5]. In the region $q^{2}<4 m_{\mu}^{2}$, where only the $e^{+} e^{-}$modes are allowed, there is a large enhancement due to the $1 / q^{2}$ scaling of the photon penguin contribution [96]. In order to remove the phase space effects in the ratio $R_{\phi}^{e \mu}$ and keep consistent with other analysis [5], we here also use the lower cut of $4 m_{\mu}^{2}$ for 

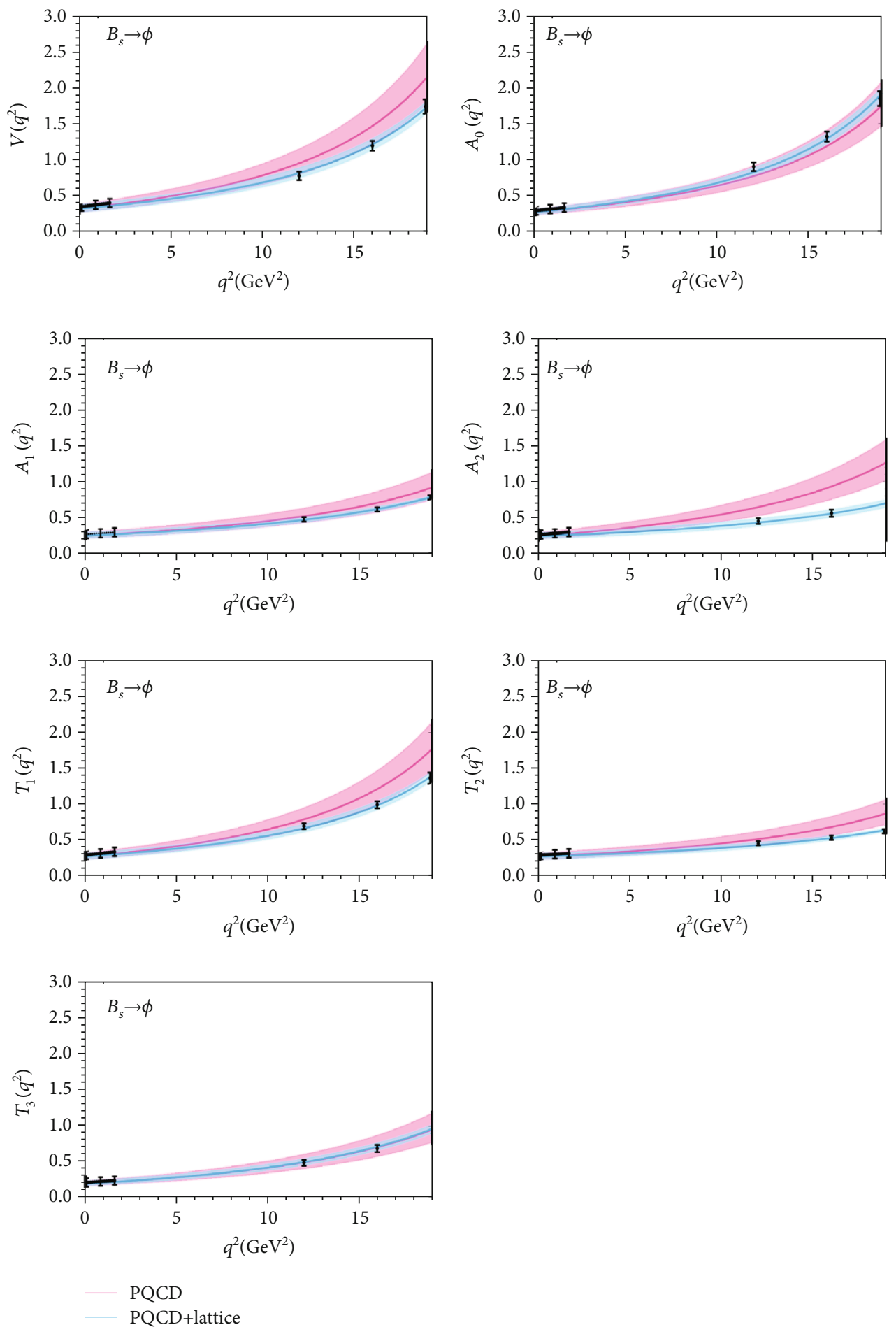

FIgURE 1: Theoretical predictions of the relevant form factors for $B_{s} \longrightarrow \phi$ transition in the PQCD approach (red curves) and the "PQCD +Lattice" approach (blue curves). The red (blue) shaded band represents the theory uncertainties. The black error bars in the low- $q^{2}$ region correspond to the PQCD predictions. The error bars in high- $q^{2}$ region are the lattice QCD results. 

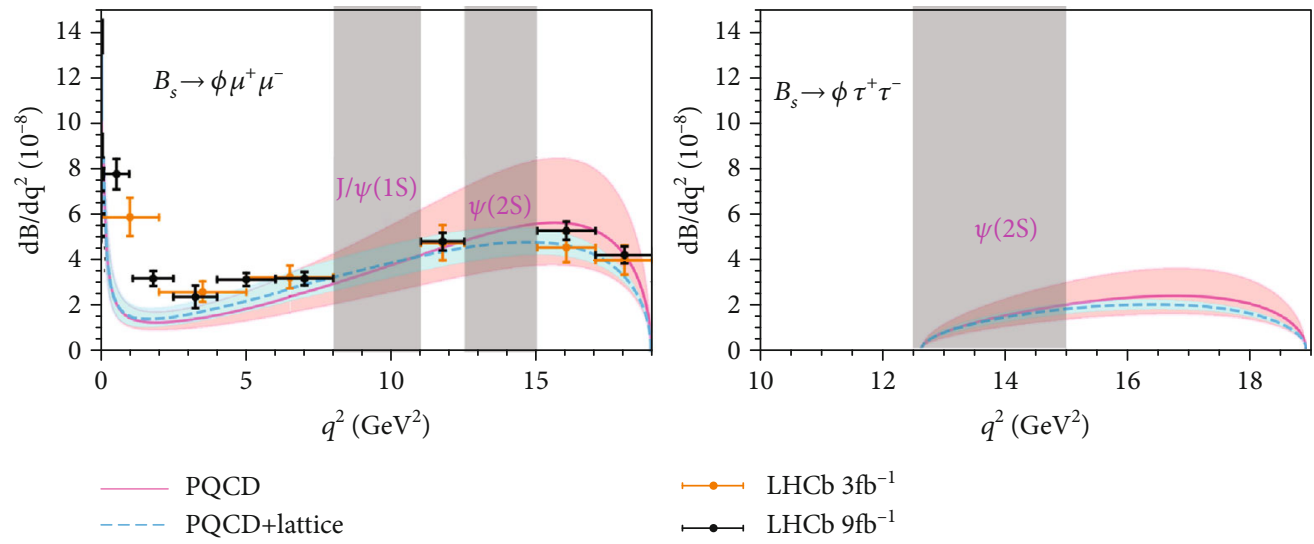

Figure 2: Theoretical predictions for the $q^{2}$-dependence of differential branching fraction $d B / d q^{2}$ for the semileptonic decays $B_{s} \longrightarrow \phi \mu^{+} \mu^{-}$ in the PQCD (red band) and "PQCD+Lattice" (blue band) approach, respectively. The crosses show the LHCb measurements in different bins as given in Reference [31] (orange ones) and in Reference [33] (black ones). The vertical grey blocks are the experimental veto regions.

TABLE 6: Theoretical predictions for the total branching fractions $B\left(B_{s} \longrightarrow \phi \ell^{+} \ell^{-}\right)$(in units of $\left.10^{-7}\right)$ in the PQCD (the first row) and "PQCD +Lattice" (the second row) approaches. As a comparison, we also list the LHCb measured value for muon channel corresponding to an integrated luminosity of $3 \mathrm{fb}^{-1}$ [31] and $9 \mathrm{fb}^{-1}$ [33] and the QCDSR predictions for all three channels [87].

\begin{tabular}{|c|c|c|c|}
\hline $\mathrm{BFs}$ & PQCD/“PQCD+Lattice” & QCDSR [87] & $\mathrm{LHCb}$ \\
\hline \multirow{2}{*}{$B\left(B_{s} \longrightarrow \phi e^{+} e^{-}\right)$} & $8.55_{-2.69}^{+4.02}(\mathrm{FFs}) \pm 0.15(\mu) \pm 0.42\left(V_{t b}\right) \pm 0.65\left(V_{t s}\right)$ & $7.12 \pm 1.40$ & \\
\hline & $8.24_{-1.53}^{+2.03}(\mathrm{FFs}) \pm 0.14(\mu) \pm 0.41\left(V_{t b}\right) \pm 0.63\left(V_{t s}\right)$ & & \\
\hline \multirow{2}{*}{$B\left(B_{s} \longrightarrow \phi \mu^{+} \mu^{-}\right)$} & $7.07_{-2.25}^{+3.37}(\mathrm{FFs}) \pm 0.12(\mu) \pm 0.38\left(V_{t b}\right) \pm 0.53\left(V_{t s}\right)$ & $7.06 \pm 1.59$ & $7.97_{-0.80}^{+0.81}[31]$ \\
\hline & $6.76_{-1.09}^{+1.39}(\mathrm{FFs}) \pm 0.11(\mu) \pm 0.33\left(V_{t b}\right) \pm 0.52\left(V_{t s}\right)$ & & $8.14 \pm 0.47[33]$ \\
\hline \multirow{2}{*}{$B\left(B_{s} \longrightarrow \phi \tau^{+} \tau^{-}\right)$} & $0.81_{-0.27}^{+0.42}(\mathrm{FFs}) \pm 0.02(\mu) \pm 0.04\left(V_{t b}\right) \pm 0.06\left(V_{t s}\right)$ & $0.35 \pm 0.17$ & \\
\hline & $0.68_{-0.06}^{+0.06}(\mathrm{FFs}) \pm 0.02(\mu) \pm 0.03\left(V_{t b}\right) \pm 0.05\left(V_{t s}\right)$ & & \\
\hline
\end{tabular}

both the electron and muon modes in the definition of the ratio $R_{\phi}^{e \mu}$ as in Reference [5]:

$$
\begin{aligned}
R_{\phi}^{e \mu} & =\frac{\int_{4 m_{\mu}^{2}}^{q_{\max }^{2}} d q^{2}\left(d B\left(B_{s} \longrightarrow \phi \mu^{+} \mu^{-}\right) / d q^{2}\right)}{\int_{4 m_{\mu}^{2}}^{q_{\max }^{2}} d q^{2}\left(d B\left(B_{s} \longrightarrow \phi e^{+} e^{-}\right) / d q^{2}\right)} \\
& = \begin{cases}0.992 \pm 0.002, & \text { in PQCD }, \\
0.991 \pm 0.002, & \text { in PQCD + Lattice }\end{cases}
\end{aligned}
$$

For the case of the ratio $R_{\phi}^{\mu \tau}$, we have

$$
\begin{aligned}
R_{\phi}^{\mu \tau} & =\frac{\int_{4 m_{\tau}^{2}}^{q_{\max }^{2}} d q^{2}\left(d B\left(B_{s} \longrightarrow \phi \tau^{+} \tau^{-}\right) / d q^{2}\right)}{\int_{4 m_{\mu}^{2}}^{q_{\max }^{2}} d q^{2}\left(d B\left(B_{s} \longrightarrow \phi \mu^{+} \mu^{-}\right) / d q^{2}\right)} \\
& = \begin{cases}0.115 \pm 0.004, & \text { in PQCD }, \\
0.100 \pm 0.009, & \text { in PQCD + Lattice }\end{cases}
\end{aligned}
$$

where the total error is the combination of the individual errors in quadrature. We suggest the $\mathrm{LHCb}$ and Belle-II to measure these two ratios.
For $B_{s} \longrightarrow \phi \mu^{+} \mu^{-}$decay, we show our theoretical predictions for the $q^{2}$-dependence of the longitudinal polarization $F_{L}\left(q^{2}\right)$, the CP-averaged angular coefficients $S_{3,4,7}\left(q^{2}\right)$, and the CP asymmetry angular coefficients $A_{5,6,8,9}\left(q^{2}\right)$ in Figure 3. As a comparison, the currently available $\mathrm{LHCb}$ measurements for these observables of $B_{s} \longrightarrow \phi \mu^{+} \mu^{-}$decay in the six $q^{2}$ bins [31] are also shown by those crosses explicitly. One can see from Figure 3 that

(1) For the longitudinal polarization $F_{L}\left(q^{2}\right)$, although both PQCD and "PQCD+Lattice" predictions all agree well with the $\mathrm{LHCb}$ measurements in the six bins, our theoretical predictions in the region of the fourth and fifth bin are little larger than the measured ones

(2) For the CP-averaged angular coefficients $S_{3,4,7}\left(q^{2}\right)$, the PQCD and "PQCD+Lattice" predictions agree very well with each other and are consistent with the $\mathrm{LHCb}$ results within the still large experimental errors. For the last two high- $q^{2}$ bins, the $\mathrm{LHCb}$ results of $S_{3}\left(S_{7}\right)$ are a little larger (smaller) than our theoretical predictions 

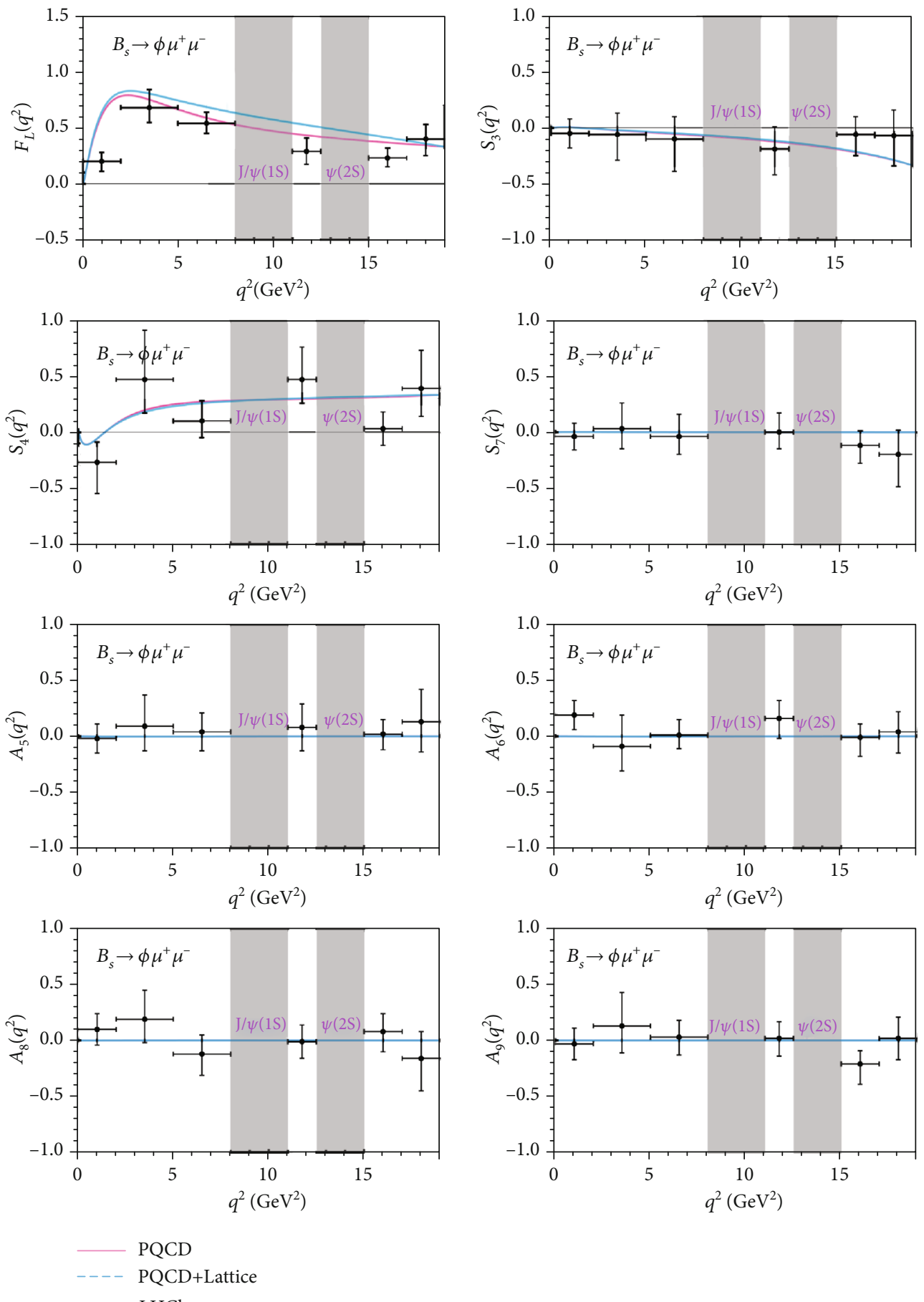

Figure 3: Theoretical predictions for the $q^{2}$-dependence of the observables $F_{L}\left(q^{2}\right), S_{3,4,7}\left(q^{2}\right)$, and $A_{5,6,8,9}\left(q^{2}\right)$ for the decay $B_{s} \longrightarrow \phi \mu^{+} \mu^{-}$in the PQCD (red lines) and "PQCD+Lattice" (blue lines) approach. The crosses in each figure represent the LHCb measurements in six $q^{2}$ bins [31]. The vertical grey blocks are the two experimental veto regions. 

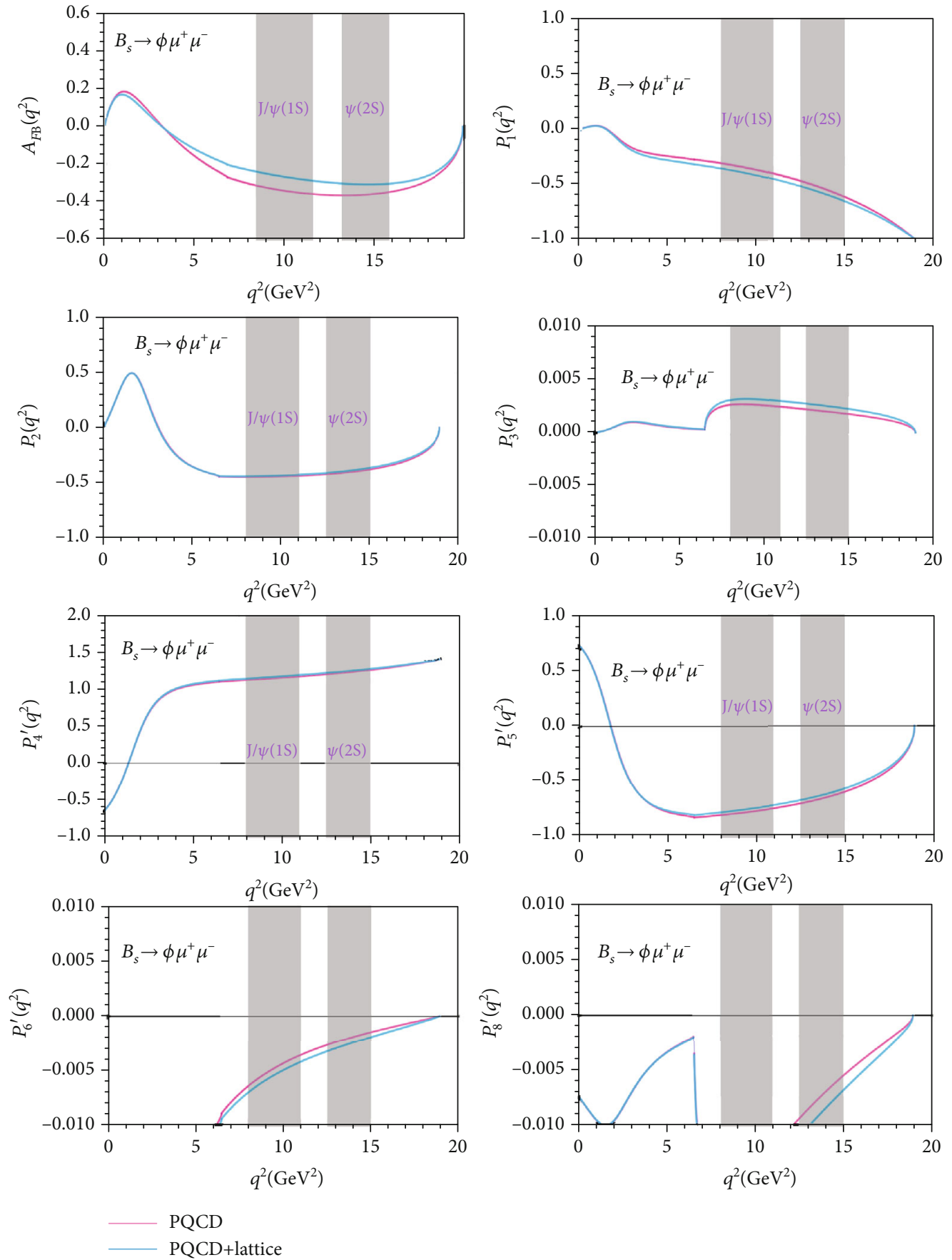

PQCD+lattice

FIgURE 4: Theoretical predictions for the $q^{2}$-dependence of the observables $A_{\mathrm{FB}}\left(q^{2}\right), P_{1,2,3}\left(q^{2}\right)$, and $P_{4,5,6,8}\left(q^{2}\right)$ for $B_{s} \longrightarrow \phi \mu^{+} \mu^{-}$decay in the PQCD (red lines) and "PQCD+Lattice" (blue lines) approach. The vertical grey blocks are the two experimental veto regions. 
TABLE 7: Theoretical predictions for the observables $F_{L}^{\phi}, A_{\mathrm{FB}}, S_{3,4,7}$, $A_{5,6,8,9}, P_{1,2,3}$, and $P_{4,5,6,8}$ integrated over the whole kinematic region for $B_{s} \longrightarrow \phi \ell^{+} \ell^{-}$decays in the PQCD (the first row) and "PQCD +Lattice" (the second row) approaches, respectively.

\begin{tabular}{|c|c|c|c|}
\hline Obs. & $\ell=e$ & $\ell=\mu$ & $\ell=\tau$ \\
\hline \multirow{2}{*}{$F_{L}^{\phi}$} & $0.383_{-0.004}^{+0.003}$ & $0.454_{-0.007}^{+0.006}$ & $0.396_{-0.003}^{+0.002}$ \\
\hline & $0.446_{-0.013}^{+0.012}$ & $0.533_{-0.001}^{+0.001}$ & $0.442_{-0.002}^{+0.002}$ \\
\hline \multirow{2}{*}{$-S_{3}$} & $0.120_{-0.004}^{+0.005}$ & $0.144_{-0.005}^{+0.005}$ & $0.080_{-0.001}^{+0.001}$ \\
\hline & $0.102_{-0.008}^{+0.009}$ & $0.124_{-0.006}^{+0.007}$ & $0.075_{-0.001}^{+0.001}$ \\
\hline \multirow{2}{*}{$S_{4}$} & $0.210_{-0.004}^{+0.004}$ & $0.258_{-0.004}^{+0.003}$ & $0.100_{-0.001}^{+0.001}$ \\
\hline & $0.201_{-0.013}^{+0.012}$ & $0.249_{-0.006}^{+0.007}$ & $0.098_{-0.001}^{+0.001}$ \\
\hline \multirow{2}{*}{$10^{3} S_{7}$} & $0.350_{-0.011}^{+0.010}$ & $0.371_{-0.013}^{+0.012}$ & $0.022_{-0.000}^{+0.001}$ \\
\hline & $0.392_{-0.024}^{+0.026}$ & $0.423_{-0.035}^{+0.040}$ & $0.030_{-0.000}^{+0.001}$ \\
\hline \multirow{2}{*}{$-10^{3} A_{5}$} & $0.489_{-0.009}^{+0.010}$ & $0.585_{-0.013}^{+0.012}$ & $0.040_{-0.000}^{+0.000}$ \\
\hline & $0.518_{-0.020}^{+0.020}$ & $0.623_{-0.046}^{+0.041}$ & $0.038_{-0.001}^{+0.001}$ \\
\hline \multirow{2}{*}{$-10^{3} A_{6}$} & $0.482_{-0.001}^{+0.001}$ & $0.577_{-0.004}^{+0.002}$ & $0.068_{-0.001}^{+0.000}$ \\
\hline & $0.429_{-0.021}^{+0.020}$ & $0.518_{-0.043}^{+0.038}$ & $0.058_{-0.001}^{+0.001}$ \\
\hline \multirow{2}{*}{$10^{4} A_{8}$} & $0.541_{-0.027}^{+0.030}$ & $0.241_{-0.020}^{+0.017}$ & $0.011_{-0.000}^{+0.000}$ \\
\hline & $0.550_{-0.095}^{+0.088}$ & $0.237_{-0.070}^{+0.060}$ & $0.014_{-0.000}^{+0.000}$ \\
\hline \multirow{2}{*}{$10^{4} A_{9}$} & $0.040_{-0.004}^{+0.002}$ & $0.054_{-0.004}^{+0.002}$ & $0.013_{-0.001}^{+0.001}$ \\
\hline & $0.041_{-0.008}^{+0.006}$ & $0.056_{-0.007}^{+0.004}$ & $0.015_{-0.001}^{+0.001}$ \\
\hline \multirow{2}{*}{$-A_{\mathrm{FB}}$} & $0.192_{-0.004}^{+0.003}$ & $0.233_{-0.004}^{+0.004}$ & $0.173_{-0.002}^{+0.002}$ \\
\hline & $0.152_{-0.008}^{+0.010}$ & $0.186_{-0.004}^{+0.005}$ & $0.151_{-0.003}^{+0.002}$ \\
\hline \multirow{2}{*}{$-P_{1}$} & $0.399_{-0.011}^{+0.014}$ & $0.555_{-0.012}^{+0.010}$ & $0.795_{-0.004}^{+0.007}$ \\
\hline & $0.381_{-0.039}^{+0.041}$ & $0.564_{-0.024}^{+0.026}$ & $0.817_{-0.005}^{+0.007}$ \\
\hline \multirow{2}{*}{$-P_{2}$} & $0.213_{-0.003}^{+0.002}$ & $0.299_{-0.001}^{+0.001}$ & $0.281_{-0.004}^{+0.003}$ \\
\hline & $0.189_{-0.013}^{+0.015}$ & $0.282_{-0.004}^{+0.005}$ & $0.268_{-0.004}^{+0.004}$ \\
\hline \multirow{2}{*}{$10^{2} P_{3}$} & $0.103_{-0.006}^{+0.003}$ & $0.142_{-0.007}^{+0.002}$ & $0.122_{-0.006}^{+0.003}$ \\
\hline & $0.118_{-0.012}^{+0.012}$ & $0.174_{-0.008}^{+0.007}$ & $0.158_{-0.003}^{+0.002}$ \\
\hline \multirow{2}{*}{$P_{4}^{\prime}$} & $1.049_{-0.015}^{+0.013}$ & $1.111_{-0.012}^{+0.011}$ & $1.338_{-0.002}^{+0.002}$ \\
\hline & $1.033_{-0.037}^{+0.033}$ & $1.098_{-0.027}^{+0.024}$ & $1.345_{-0.003}^{+0.002}$ \\
\hline \multirow{2}{*}{$-P^{\prime}{ }_{5}$} & $0.482_{-0.004}^{+0.002}$ & $0.524_{-0.005}^{+0.003}$ & $0.427_{-0.006}^{+0.005}$ \\
\hline & $0.470_{-0.010}^{+0.008}$ & $0.515_{-0.003}^{+0.002}$ & $0.408_{-0.006}^{+0.006}$ \\
\hline \multirow{2}{*}{$-10^{3} P_{6}^{\prime}$} & $0.875_{-0.030}^{+0.031}$ & $0.780_{-0.029}^{+0.028}$ & $0.069_{-0.001}^{+0.001}$ \\
\hline & $1.007_{-0.101}^{+0.089}$ & $0.910_{-0.089}^{+0.078}$ & $0.097_{-0.001}^{+0.001}$ \\
\hline \multirow{2}{*}{$-10^{2} P_{8}^{\prime}$} & $0.643_{-0.003}^{+0.009}$ & $0.640_{-0.008}^{+0.004}$ & $0.287_{-0.002}^{+0.002}$ \\
\hline & $0.749_{-0.004}^{+0.004}$ & $0.748_{-0.006}^{+0.006}$ & $0.374_{-0.001}^{+0.001}$ \\
\hline
\end{tabular}

(3) For the CP asymmetry angular coefficients $A_{5,6,8,9}$ $\left(q^{2}\right)$, the PQCD and "PQCD+Lattice" predictions are very small: in the range of $10^{-4}$ to $10^{-2}$. For the $\mathrm{LHCb}$ measurements in the six bins, they are clearly consistent with our theoretical predictions due to still large experimental errors
In Figure 4, we show our theoretical predictions for the $q^{2}$ -dependence of the forward-backward asymmetry $A_{\mathrm{FB}}\left(q^{2}\right)$, the optimized observables $P_{1,2,3}\left(q^{2}\right)$, and $P_{4,5,6,8}\left(q^{2}\right)$ for $B_{s}$ $\longrightarrow \phi \mu^{+} \mu^{-}$decay. Unfortunately, there exist no any experimental measurements for these observables. We have to wait for future $\mathrm{LHCb}$ and Belle-II measurements. Analogous to Figure 3, the vertical grey blocks in Figure 4 also denote the two experimental veto regions of $q^{2}: 8.0<q^{2}<11.0 \mathrm{GeV}^{2}$ and $12.5<q^{2}<15.0 \mathrm{GeV}^{2}$.

In Table 7, we list the theoretical predictions for the values of the observables $F_{L}^{\phi}, A_{\mathrm{FB}}, S_{3,4,7}, A_{5,6,8,9}, P_{1,2,3}$, and $P_{4,5,6,8}$, obtained after the integrations over the whole kinematic region of $q^{2}$ for the semileptonic decays $B_{s} \longrightarrow \phi \ell^{+} \ell^{-}$ with $\ell=(e, \mu, \tau)$ in the PQCD (the first row) and "PQCD +Lattice" (the second row) approaches, respectively. Of course, the regions corresponding to resonance $J / \psi(1 S)$ and $\psi(2 S)$, say $8.0<q^{2}<11.0 \mathrm{GeV}^{2}$ and $12.5<q^{2}<15.0$ $\mathrm{GeV}^{2}$ numerically, are also cut off here. The total errors are the combinations of the individual errors from the form factors, the renormalization scales, and the relevant CKM matrix elements. The above theoretical predictions should be tested in the near future LHCb and Belle-II experiments. For the considered $B_{s}$ meson decays, one should consider the effects from the $B_{s}-\bar{B}_{s}$ mixing [6]. The theoretical framework for examining the timedependent decays with the inclusion of such mixing effects can be found in Reference [97]. The authors of Reference [97] proved that the mixing effects on the values of decay rates and $\mathrm{CP}$-averaged observables are generally within a few percent and could be neglected.

4.3. The $q^{2}$-Binned Predictions. For $B_{s} \longrightarrow \phi \mu^{+} \mu^{-}$decay mode, the LHCb Collaboration has reported their experimental measurements for many physical observables in several $q^{2}$ bins $[31,33]$. In order to compare our theoretical predictions with the $\mathrm{LHCb}$ results bin by bin, we make the same choices of the $q^{2}$ bins as LHCb did, calculate, and show our theoretical predictions for the branching ratio $B\left(B_{s}\right.$ $\left.\longrightarrow \phi \ell^{+} \ell^{-}\right)$and the asymmetry $F_{L}^{\phi}$ with $\ell=(\mu, \tau)$ in Tables 8 and 9 and the observables $S_{3,4,7}$ with $\ell=\mu$ in Table 10. For observables $S_{7}$ and $A_{5,6,8,9}$, in fact, our theoretical predictions for their values are very small, say in the range of $10^{-3}-10^{-4}$ in magnitude, but still agree with the LHCb measurements in different bins [31] due to still large experimental errors. For the observables $P_{3}$ and $P_{6,8}^{\prime}$, they are also very small in size: in the range of $10^{-3}-10^{-4}$ and there exist no corresponding data at present. For observables $P_{1,2}$ and $P_{4,5}^{\prime}$, on the other hand, although there exist no experimental measurements for them at present, they are relatively large in size and may be measured in the near future $\mathrm{LHCb}$ and Belle-II experiments, so we calculate and list the theoretical predictions of these observables bin by bin for the cases of $\ell=(\mu, \tau)$ in Tables 11 and 12. Very recently, $\mathrm{LHCb}$ reported some new measurements for the angular observables of $B_{s} \longrightarrow \phi \mu^{+} \mu^{-}$decay [34] in the $q^{2}$ bins different from those in their previous work [31], which will be studied in our next work. 
TABLE 8: Theoretical predictions for the $q^{2}$-binned observables $\mathscr{B}\left(B_{s} \longrightarrow \phi \mu^{+} \mu^{-}\right)$(in unit of $\left.10^{-7}\right)$ in the PQCD (the first low) and "PQCD +Lattice" (the second row) approach, respectively. For a comparison, we also list the new LHCb measurements [33].

\begin{tabular}{|c|c|c|c|c|c|}
\hline$q^{2}$ bin $\left(\mathrm{GeV}^{2}\right)$ & $\mathscr{B}(\ell=\mu)$ & LHCb [33] & $q^{2}$ bin $\left(\mathrm{GeV}^{2}\right)$ & $\mathscr{B}(\ell=\mu)$ & $\mathrm{LHCb}[33]$ \\
\hline \multirow{2}{*}[0.10,0.98]{} & $0.25_{-0.07}^{+0.10}$ & \multirow{2}{*}{$0.68 \pm 0.06$} & \multirow{2}{*}[1.10,2.50]{} & $0.19_{-0.05}^{+0.08}$ & \multirow{2}{*}{$0.44 \pm 0.05$} \\
\hline & $0.25_{-0.07}^{+0.10}$ & & & $0.21_{-0.05}^{+0.07}$ & \\
\hline \multirow{2}{*}[2.50,4.00]{} & $0.23_{-0.07}^{+0.09}$ & \multirow{2}{*}{$0.35 \pm 0.04$} & \multirow{2}{*}[4.00,6.00]{} & $0.41_{-0.12}^{+0.18}$ & \multirow{2}{*}{$0.62 \pm 0.06$} \\
\hline & $0.27_{-0.06}^{+0.09}$ & & & $0.50_{-0.11}^{+0.14}$ & \\
\hline \multirow{2}{*}[6.00,8.00]{} & $0.61_{-0.19}^{+0.28}$ & \multirow{2}{*}{$0.63 \pm 0.06$} & \multirow{2}{*}[11.0,12.5]{} & $0.78_{-0.25}^{+0.38}$ & \multirow{2}{*}{$0.72 \pm 0.06$} \\
\hline & $0.69_{-0.13}^{+0.18}$ & & & $0.76_{-0.11}^{+0.14}$ & \\
\hline \multirow{2}{*}[15.00,17.00]{} & $1.26_{-0.42}^{+0.64}$ & \multirow{2}{*}{$1.05 \pm 0.08$} & \multirow{2}{*}[17.0,19.0]{} & $0.86_{-0.30}^{+0.45}$ & \multirow{2}{*}{$0.84 \pm 0.07$} \\
\hline & $1.04_{-0.10}^{+0.11}$ & & & $0.67_{-0.06}^{+0.05}$ & \\
\hline \multirow{2}{*}[1.10,6.00]{} & $0.83_{-0.24}^{+0.35}$ & \multirow{2}{*}{$1.41 \pm 0.10$} & \multirow{2}{*}[15.0,19.0]{} & $2.12_{-0.71}^{+1.09}$ & \multirow{2}{*}{$1.85 \pm 0.13$} \\
\hline & $0.98_{-0.22}^{+0.30}$ & & & $1.70_{-0.16}^{+0.17}$ & \\
\hline
\end{tabular}

The definitions of the $q^{2}$-binned observables are the following:

$$
\begin{aligned}
& B\left(q_{1}^{2}, q_{2}^{2}\right)=\int_{q_{1}^{2}}^{q_{2}^{2}} d q^{2} \frac{d B\left(B_{s} \longrightarrow \phi \ell^{+} \ell^{-}\right)}{d q^{2}}, \\
& F_{L}^{\phi}\left(q_{1}^{2}, q_{2}^{2}\right)=\frac{\int_{q_{1}^{2}}^{q_{2}^{2}} d q^{2}\left[3\left(I_{1}^{c}+\bar{I}_{1}^{c}\right)-\left(I_{2}^{c}+\bar{I}_{2}^{c}\right)\right]}{4 \int_{q_{1}^{2}}^{q_{2}^{2}} d q^{2}\left[d(\Gamma+\bar{\Gamma}) / d q^{2}\right]}, \\
& A_{\mathrm{FB}}\left(q_{1}^{2}, q_{2}^{2}\right)=\frac{3 \int_{q_{1}^{2}}^{q_{2}^{2}} d q^{2}\left(I_{6}^{s}+\bar{I}_{6}^{s}\right)}{4 \int_{q_{1}^{2}}^{q_{2}^{2}} d q^{2}\left[d(\Gamma+\bar{\Gamma}) / d q^{2}\right]}, \\
& S_{3,4,7}\left(q_{1}^{2}, q_{2}^{2}\right)=\frac{\int_{q_{1}^{2}}^{q_{2}^{2}} d q^{2}\left(I_{3,4,7}+\bar{I}_{3,4,7}\right)}{\int_{q_{1}^{2}}^{q_{2}^{2}} d q^{2}\left[d(\Gamma+\bar{\Gamma}) / d q^{2}\right]}, \\
& P_{1}\left(q_{1}^{2}, q_{2}^{2}\right)=\frac{\int_{q_{1}^{2}}^{q_{2}^{2}} d q^{2}\left(S_{3}\right)}{2 \int_{q_{1}^{2}}^{q_{2}^{2}} d q^{2}\left(S_{2}^{s}\right)} \\
& P_{2}\left(q_{1}^{2}, q_{2}^{2}\right)=\frac{\int_{q_{1}^{2}}^{q_{2}^{2}} d q^{2}\left(\beta_{\ell} S_{6}^{s}\right)}{8 \int_{q_{1}^{2}}^{q_{2}^{2}} d q^{2}\left(S_{2}^{s}\right)} \\
& P_{4}^{\prime}\left(q_{1}^{2}, q_{2}^{2}\right)=\frac{\int_{q_{1}^{2}}^{q_{2}^{2}} d q^{2}\left(S_{4}\right)}{\sqrt{-\int_{q_{1}^{2}}^{q_{2}^{2}} d q^{2}\left(S_{2}^{c} S_{2}^{s}\right)}}, \\
& P_{5}^{\prime}\left(q_{1}^{2}, q_{2}^{2}\right)=\frac{\int_{q_{1}^{2}}^{q_{2}^{2}} d q^{2}\left(\beta_{\ell} S_{5}\right)}{2 \sqrt{-\int_{q_{1}^{2}}^{q_{2}^{2}} d q^{2}\left(S_{2}^{c} S_{2}^{s}\right)}} .
\end{aligned}
$$

From the numerical values as shown in Figure 3 and in Tables 8-10, we find the following points about the relevant physical observables of the considered $B_{s} \longrightarrow \phi \mu^{+} \mu^{-}$decays in bins:
(1) For $B_{s} \longrightarrow \phi \mu^{+} \mu^{-}$decay, besides the good consistency between the theory and the LHCb data for the integrated total branching ratio $B\left(B_{s} \longrightarrow \phi \mu^{+}\right.$ $\mu^{-}$) as listed in Equation (50), the PQCD and "PQCD+Lattice" predictions for $B\left(B_{s} \longrightarrow \phi \mu^{+} \mu^{-}\right)$ in most bins do agree well with the measured ones within $2 \sigma$ errors. For the first low- $q^{2}$ bin $0.1<q^{2}$ $<2\left(\mathrm{GeV}^{2}\right)$, however, the central value of the $\mathrm{LHCb}$ result $1.11 \pm 0.16$ is larger than the theoretical ones by roughly a factor of three. The $\mathrm{LHCb}$ results of $B\left(B_{s} \longrightarrow \phi \mu^{+} \mu^{-}\right)$in different bins of $q^{2}$ as listed in the third column of Table 9 are obtained from the results as given in References $[31,33]$ by multiplying the LHCb measured values of differential decay rate $d B\left(B_{s} \longrightarrow \phi \mu^{+} \mu^{-}\right) / d q^{2}$ with the width of the corresponding bin $\left(q_{2}^{2}-q_{1}^{2}\right)$. The theoretical errors of our theoretical predictions of the branching ratios in bins are still relatively large, while the differences between the PQCD and "PQCD+Lattice" predictions for $B\left(B_{s} \longrightarrow \phi \ell^{+}\right.$ $\left.\ell^{-}\right)$with $\ell=(\mu, \tau)$ are small

(2) In the first low- $q^{2}$ bin $0.1<q^{2}<2\left(\mathrm{GeV}^{2}\right)$, both the PQCD and "PQCD+Lattice" predictions for $F_{\mathrm{L}}^{\phi}(\ell=$ $\mu)$ are larger than the $\mathrm{LHCb}$ measured results $\left.F_{L}^{\phi}(\ell=\mu)\right|_{\mathrm{LHCb}}=0.20_{-0.09}^{+0.08} \pm 0.02$ [31]. For other bins, both PQCD and "PQCD+Lattice" predictions of $F_{L}^{\phi}$ for muon mode do agree very well with currently available $\mathrm{LHCb}$ measured values [31] within $2 \sigma$ errors. It is worth of remaining that our theoretical predictions of $F_{L}^{\phi}$ have a little error of $\sim 2 \%$ due to the strong cancellation of the theoretical errors in the ratios. The theoretical predictions for $B\left(B_{s} \longrightarrow\right.$ $\left.\phi \tau^{+} \tau^{-}\right)$and $F_{L}^{\phi}(\ell=\tau)$ in different bins of $q^{2}$ as listed in Table 9 will be tested by future experimental measurements

(3) For the observables $S_{3,4,7}$, as listed in Table 10, the PQCD and "PQCD+Lattice" predictions for their 
TABle 9: Theoretical predictions for the $q^{2}$-binned observables $\mathscr{B}\left(B_{s} \longrightarrow \phi \ell^{+} \ell^{-}\right)$(in unit of $\left.10^{-7}\right)$ in the PQCD (the first low) and "PQCD +Lattice" (the second row) approach, respectively. For a comparison, we also list the new LHCb measurements [33].

\begin{tabular}{|c|c|c|c|c|c|c|}
\hline$q^{2} \operatorname{bin}\left(\mathrm{GeV}^{2}\right)$ & $\mathscr{B}(l=\mu)$ & $\mathrm{LHCb}$ & $\mathscr{B}(l=\tau)$ & $F_{L}^{\phi}(l=\mu)$ & $\mathrm{LHCb}$ & $F_{L}^{\phi}(l=\tau)$ \\
\hline \multirow{2}{*}[0.1,2.0]{} & $0.39_{-0.11}^{+0.16}$ & $1.11 \pm 0.16[31]$ & - & $0.441_{-0.008}^{+0.007}$ & $0.20 \pm 0.09$ & - \\
\hline & $0.40_{-0.11}^{+0.16}$ & & - & $0.472_{-0.012}^{+0.011}$ & & - \\
\hline \multirow{2}{*}[2.0,5.0]{} & $0.48_{-0.14}^{+0.20}$ & $0.77 \pm 0.14$ & - & $0.738_{-0.009}^{+0.008}$ & $0.68 \pm 0.15$ & - \\
\hline & $0.58_{-0.13}^{+0.18}$ & & - & $0.796_{-0.007}^{+0.007}$ & & - \\
\hline \multirow{2}{*}[5.0,8.0]{} & $0.84_{-0.25}^{+0.37}$ & $0.96 \pm 0.15[31]$ & - & $0.584_{-0.009}^{+0.008}$ & $0.54 \pm 0.10$ & - \\
\hline & $0.96_{-0.19}^{+0.25}$ & & - & $0.682_{-0.008}^{+0.010}$ & & - \\
\hline \multirow{2}{*}[11.0,12.5]{} & $0.78_{-0.25}^{+0.38}$ & $0.71 \pm 0.12[31]$ & - & $0.433_{-0.005}^{+0.003}$ & $0.29 \pm 0.11$ & - \\
\hline & $0.76_{-0.11}^{+0.14}$ & $0.72 \pm 0.06$ & - & $0.524_{-0.008}^{+0.008}$ & & - \\
\hline \multirow{2}{*}[15.0,17.0]{} & $1.12_{-0.40}^{+0.61}$ & $0.90 \pm 0.13[31]$ & $0.45_{-0.14}^{+0.22}$ & $0.368_{-0.001}^{+0.001}$ & $0.23 \pm 0.09$ & $0.421_{-0.004}^{+0.003}$ \\
\hline & $0.99_{-0.10}^{+0.11}$ & $1.05 \pm 0.08$ & $0.39_{-0.04}^{+0.04}$ & $0.412_{-0.004}^{+0.004}$ & & $0.479_{-0.003}^{+0.003}$ \\
\hline \multirow{2}{*}[17.0,19.0]{} & $0.80_{-0.27}^{+0.41}$ & $0.75 \pm 0.13[31]$ & $0.36_{-0.12}^{+0.18}$ & $0.346_{-0.001}^{+0.001}$ & $0.40 \pm 0.14$ & $0.365_{-0.002}^{+0.001}$ \\
\hline & $0.67_{-0.05}^{+0.05}$ & $0.82 \pm 0.07$ & $0.29_{-0.02}^{+0.02}$ & $0.363_{-0.001}^{+0.001}$ & & $0.391_{-0.001}^{+0.001}$ \\
\hline \multirow{2}{*}[1.0,6.0]{} & $0.93_{-0.27}^{+0.29}$ & $1.29 \pm 0.19[31]$ & - & $0.708_{-0.009}^{+0.007}$ & $0.63 \pm 0.09$ & - \\
\hline & $1.10_{-0.25}^{+0.34}$ & & - & $0.777_{-0.006}^{+0.008}$ & & - \\
\hline \multirow{2}{*}[15.0,19.0]{} & $1.99_{-0.71}^{+1.02}$ & $1.62 \pm 0.20[31]$ & $0.82_{-0.26}^{+0.40}$ & $0.359_{-0.001}^{+0.002}$ & $0.29 \pm 0.07$ & $0.396_{-0.003}^{+0.003}$ \\
\hline & $1.60_{-0.16}^{+0.16}$ & $1.85 \pm 0.13$ & $0.68_{-0.06}^{+0.06}$ & $0.394_{-0.003}^{+0.003}$ & & $0.442_{-0.001}^{+0.002}$ \\
\hline
\end{tabular}

TABLE 10: Theoretical predictions for the $q^{2}$-binned observables $S_{3,4,7}$ of the decays $B_{s} \longrightarrow \phi \mu^{+} \mu^{-}$in the PQCD (the first low) and "PQCD +Lattice" (the second row) approaches. For a comparison, we also list the LHCb measured values [31].

\begin{tabular}{|c|c|c|c|c|c|c|}
\hline \multirow[b]{2}{*}{$q^{2}$ bin $\left(\mathrm{GeV}^{2}\right)$} & \multicolumn{2}{|c|}{$S_{3}$} & \multicolumn{2}{|c|}{$S_{4}$} & \multicolumn{2}{|c|}{$S_{7}$} \\
\hline & Theor. & $\mathrm{LHCb}$ & Theor. & $\mathrm{LHCb}$ & Theor. $\left(10^{-3}\right)$ & $\mathrm{LHCb}$ \\
\hline \multirow{2}{*}[0.1,2.0]{} & $0.003_{-0.000}^{+0.000}$ & $-0.05 \pm 0.13$ & $-0.054_{-0.001}^{+0.001}$ & $-0.27 \pm 0.23$ & $1.571_{-0.002}^{+0.001}$ & $-0.04 \pm 0.12$ \\
\hline & $0.002_{-0.001}^{+0.000}$ & & $-0.053_{-0.000}^{+0.000}$ & & $1.551_{-0.006}^{+0.003}$ & \\
\hline \multirow{2}{*}[2.0,5.0]{} & $-0.024_{-0.002}^{+0.002}$ & $-0.06 \pm 0.21$ & $0.191_{-0.004}^{+0.004}$ & $0.47 \pm 0.37$ & $1.065_{-0.003}^{+0.003}$ & $0.03 \pm 0.21$ \\
\hline & $-0.021_{-0.001}^{+0.001}$ & & $0.177_{-0.003}^{+0.003}$ & & $0.979_{-0.018}^{+0.017}$ & \\
\hline \multirow{2}{*}[5.0,8.0]{} & $-0.057_{-0.003}^{+0.003}$ & $-0.10 \pm 0.25$ & $0.270_{-0.002}^{+0.002}$ & $0.10 \pm 0.17$ & $0.453_{-0.003}^{+0.003}$ & $-0.04 \pm 0.18$ \\
\hline & $-0.050_{-0.003}^{+0.004}$ & & $0.259_{-0.003}^{+0.003}$ & & $0.453_{-0.005}^{+0.004}$ & \\
\hline \multirow{2}{*}[11.0,12.5]{} & $-0.124_{-0.003}^{+0.004}$ & $-0.19 \pm 0.21$ & $0.296_{-0.001}^{+0.001}$ & $0.47 \pm 0.25$ & $0.153_{-0.002}^{+0.002}$ & $0.00 \pm 0.16$ \\
\hline & $-0.115_{-0.001}^{+0.002}$ & & $0.303_{-0.001}^{+0.001}$ & & $0.185_{-0.001}^{+0.001}$ & \\
\hline \multirow{2}{*}[15.0,17.0]{} & $-0.219_{-0.003}^{+0.003}$ & $-0.06 \pm 0.18$ & $0.314_{-0.001}^{+0.001}$ & $0.03 \pm 0.15$ & $0.052_{-0.001}^{+0.001}$ & $-0.12 \pm 0.15$ \\
\hline & $-0.213_{-0.001}^{+0.001}$ & & $0.323_{-0.001}^{+0.001}$ & & $0.071_{-0.001}^{+0.001}$ & \\
\hline \multirow{2}{*}[17.0,19.0]{} & $-0.283_{-0.001}^{+0.001}$ & $-0.07 \pm 0.25$ & $0.325_{-0.001}^{+0.001}$ & $0.39 \pm 0.30$ & $0.019_{-0.001}^{+0.001}$ & $-0.20 \pm 0.26$ \\
\hline & $-0.281_{-0.001}^{+0.001}$ & & $0.329_{-0.001}^{+0.001}$ & & $0.027_{-0.001}^{+0.001}$ & \\
\hline \multirow{2}{*}[1.0,6.0]{} & $-0.026_{-0.002}^{+0.002}$ & $-0.02 \pm 0.13$ & $0.180_{-0.004}^{+0.004}$ & $0.19 \pm 0.14$ & $1.063_{-0.001}^{+0.001}$ & $0.03 \pm 0.14$ \\
\hline & $-0.023_{-0.001}^{+0.001}$ & & $0.169_{-0.001}^{+0.001}$ & & $0.985_{-0.022}^{+0.020}$ & \\
\hline \multirow{2}{*}[15.0,19.0]{} & $-0.245_{-0.002}^{+0.002}$ & $-0.09 \pm 0.12$ & $0.318_{-0.001}^{+0.001}$ & $0.14 \pm 0.11$ & $0.038_{-0.002}^{+0.002}$ & $-0.13 \pm 0.11$ \\
\hline & $-0.239_{-0.001}^{+0.001}$ & & $0.325_{-0.001}^{+0.001}$ & & $0.054_{-0.001}^{+0.001}$ & \\
\hline
\end{tabular}


TABLE 11: Theoretical predictions for the $q^{2}$-binned observables $A_{\mathrm{FB}}^{\mu}$ and $P_{1,2}$ of the decays $B_{s} \longrightarrow \phi \ell^{+} \ell^{-}$with $\ell=(\mu, \tau)$ in the PQCD (the first low) and "PQCD+Lattice" (the second row) approaches.

\begin{tabular}{|c|c|c|c|c|c|c|}
\hline$q^{2}$ bin $\left(\mathrm{GeV}^{2}\right)$ & $A_{\mathrm{FB}}^{\mu}$ & $A_{\mathrm{FB}}^{\tau}$ & $P_{1}(\ell=\mu)$ & $P_{1}(\ell=\tau)$ & $P_{2}(\ell=\mu)$ & $P_{2}(\ell=\tau)$ \\
\hline \multirow{2}{*}[0.1,2.0]{} & $0.131_{-0.003}^{+0.003}$ & - & $0.015_{-0.001}^{+0.001}$ & - & $0.206_{-0.001}^{+0.001}$ & - \\
\hline & $0.122_{-0.002}^{+0.003}$ & - & $0.013_{-0.001}^{+0.001}$ & - & $0.204_{-0.001}^{+0.001}$ & - \\
\hline \multirow{2}{*}[2.0,5.0]{} & $-0.047_{-0.003}^{+0.004}$ & - & $-0.197_{-0.007}^{+0.007}$ & - & $-0.128_{-0.003}^{+0.003}$ & - \\
\hline & $-0.038_{-0.002}^{+0.001}$ & - & $-0.225_{-0.006}^{+0.005}$ & - & $-0.132_{-0.002}^{+0.002}$ & - \\
\hline \multirow{2}{*}[5.0,8.0]{} & $-0.265_{-0.005}^{+0.006}$ & - & $-0.280_{-0.008}^{+0.009}$ & - & $-0.431_{-0.001}^{+0.001}$ & - \\
\hline & $-0.200_{-0.006}^{+0.006}$ & - & $-0.323_{-0.004}^{+0.003}$ & - & $-0.427_{-0.001}^{+0.001}$ & - \\
\hline \multirow{2}{*}[11.0,12.5]{} & $-0.365_{-0.001}^{+0.001}$ & - & $-0.440_{-0.008}^{+0.010}$ & - & $-0.431_{-0.003}^{+0.002}$ & - \\
\hline & $-0.298_{-0.005}^{+0.005}$ & - & $-0.489_{-0.003}^{+0.002}$ & - & $-0.420_{-0.001}^{+0.001}$ & - \\
\hline \multirow{2}{*}[15.0,17.0]{} & $-0.326_{-0.001}^{+0.001}$ & $-0.188_{-0.001}^{+0.001}$ & $-0.697_{-0.006}^{+0.009}$ & $-0.707_{-0.005}^{+0.009}$ & $-0.345_{-0.004}^{+0.003}$ & $-0.341_{-0.004}^{+0.003}$ \\
\hline & $-0.290_{-0.005}^{+0.005}$ & $-0.160_{-0.003}^{+0.003}$ & $-0.730_{-0.005}^{+0.007}$ & $-0.738_{-0.006}^{+0.006}$ & $-0.330_{-0.003}^{+0.003}$ & $-0.326_{-0.004}^{+0.003}$ \\
\hline \multirow{2}{*}[17.0,19.0]{} & $-0.226_{-0.003}^{+0.002}$ & $-0.153_{-0.002}^{+0.001}$ & $-0.869_{-0.003}^{+0.005}$ & $-0.875_{-0.003}^{+0.005}$ & $-0.231_{-0.003}^{+0.002}$ & $-0.225_{-0.004}^{+0.003}$ \\
\hline & $-0.208_{-0.003}^{+0.004}$ & $-0.137_{-0.002}^{+0.003}$ & $-0.884_{-0.004}^{+0.004}$ & $-0.890_{-0.003}^{+0.005}$ & $-0.219_{-0.004}^{+0.003}$ & $-0.213_{-0.004}^{+0.004}$ \\
\hline
\end{tabular}

values in all bins are in the range of $10^{-3}-10^{-1}$ and show a good agreement with the $\mathrm{LHCb}$ measured values [31]. The errors of the theoretical predictions are also very small, $\sim 2 \%$ in magnitude, because of their nature of the ratios. In all bins, the LHCb measured values of $S_{3,4,7}$ are still consistent with zero due to their still large errors, which is a clear feature as can be seen easily from the numerical values in Table 10 and the crosses in Figure 3

In Tables 11 and 12, we show the PQCD and "PQCD +Lattice" predictions for the physical observables $A_{\mathrm{FB}}^{\mu, \tau}, P_{1,2}^{\mu, \tau}$ and $P_{4,5}^{\prime}(\ell=e, \mu, \tau)$ in six bins. These physical observables could be tested in the near future LHCb and Belle-II experiments.

\section{Summary}

In this paper, we made a systematic study of the semileptonic decays $B_{s} \longrightarrow \phi \ell^{+} \ell^{-}$with $\ell^{-}=\left(e^{-}, \mu^{-}, \tau^{-}\right)$using the PQCD and the "PQCD+Lattice" factorization approach, respectively. We first evaluated all relevant form factors in the low $-q^{2}$ region using the PQCD approach, and we also took currently available lattice QCD results at the high- $q^{2}$ points $q^{2}=(12,16,18.9) \mathrm{GeV}^{2}$ as additional input to improve the extrapolation of the form factors from the low to the high $-q^{2}$ region. We calculated the branching ratios $B$ $\left(B_{s} \longrightarrow \phi \ell^{+} \ell^{-}\right)$, the CP-averaged $\phi$ longitudinal polarization fraction $F_{L}\left(q^{2}\right)$, the forward-backward asymmetry $A_{\mathrm{FB}}\left(q^{2}\right)$, the CP-averaged angular coefficients $S_{3,4,7}\left(q^{2}\right)$, the CP asymmetry angular coefficients $A_{5,6,8,9}\left(q^{2}\right)$, the optimized observables $P_{1,2,3}\left(q^{2}\right)$, and $P_{4,5,6,8}\left(q^{2}\right)$. For $B_{s} \longrightarrow \phi \mu^{+} \mu^{-}$decay mode, we calculated the binned values of the branching ratio
$B\left(B_{s} \longrightarrow \phi \mu^{+} \mu^{-}\right)$and the observables $F_{L}^{\phi}$ and $S_{3,4,7}$ in the same bins as defined by LHCb Collaboration [31] in order to compare our theoretical predictions with those currently available $\mathrm{LHCb}$ measurements bin by bin directly.

Based on the analytical evaluations, the numerical results, and the phenomenological analysis, we found the following main points:

(1) For the branching ratio $B\left(B_{s} \longrightarrow \phi \mu^{+} \mu^{-}\right)$, the PQCD and "PQCD+Lattice" predictions are $\left(7.07_{-2.34}^{+3.43}\right) \times 1$ $0^{-7}$ and $\left(6.76_{-1.25}^{+1.52}\right) \times 10^{-7}$, respectively, which agree well with the LHCb measured values $[31,33]$ and the QCDSR prediction within still large errors. For the electron and tau mode, our theoretical predictions for their decay rates are also well consistent with the corresponding QCDSR predictions and to be tested by future experimental measurements

(2) For the ratios of the branching ratios $R_{\phi}^{e \mu}$ and $R_{\phi}^{\mu \tau}$, the PQCD and "PQCD+Lattice" predictions agree with each other and with small theoretical errors because of the strong cancellation of the theoretical errors in such ratios. We suggest the LHCb and Belle-II Collaboration to measure these ratios

(3) For the longitudinal polarization $F_{L}$, both PQCD and "PQCD+Lattice" predictions agree with the LHCb measurements in the considered bins within the errors. For the CP-averaged angular coefficients $S_{3,4,7}$, the PQCD and "PQCD+Lattice" predictions in all bins are small in magnitude, in the range of 1 $0^{-3}-10^{-1}$, and agree well with the LHCb results within the still large experimental errors. For the CP asymmetry angular coefficients $A_{5,6,8,9}$, the PQCD and "PQCD+Lattice" predictions are very 
TABLE 12: Theoretical predictions for the $q^{2}$-binned optimized observables $P^{\prime}{ }_{4}$ and $P^{\prime}{ }_{5}$ of the decays $B_{s} \longrightarrow \phi \ell^{+} \ell^{-}$with $\ell=(e, \mu, \tau)$ in the PQCD (the first low) and "PQCD+Lattice" (the second row) approach.

\begin{tabular}{lcccrrr}
\hline$q^{2}$ bin $\left(\mathrm{GeV}^{2}\right)$ & $P^{\prime}{ }_{4}(\ell=e)$ & $P^{\prime}{ }_{4}(\ell=\mu)$ & $P^{\prime}{ }_{4}(\ell=\tau)$ & $P^{\prime}{ }_{5}(\ell=e)$ & $P^{\prime}{ }_{5}(\ell=\mu)$ & $P^{\prime}(\ell=\tau)$ \\
\hline$[0.1,2.0]$ & $-0.322_{-0.005}^{+0.005}$ & $-0.289_{-0.005}^{+0.005}$ & - & $0.484_{-0.002}^{+0.001}$ & $0.459_{-0.002}^{+0.001}$ \\
& $-0.318_{-0.001}^{+0.001}$ & $-0.285_{-0.001}^{+0.001}$ & - & $0.475_{-0.001}^{+0.002}$ & $0.449_{-0.001}^{+0.001}$ \\
{$[2.0,5.0]$} & $0.903_{-0.007}^{+0.007}$ & $0.903_{-0.007}^{+0.007}$ & - & $-0.606_{-0.002}^{+0.001}$ & $-0.607_{-0.002}^{+0.001}$ & - \\
& $0.916_{-0.001}^{+0.001}$ & $0.916_{-0.001}^{+0.001}$ & - & $-0.608_{-0.003}^{+0.004}$ & $-0.608_{-0.004}^{+0.004}$ & - \\
{$[5.0,8.0]$} & $1.110_{-0.005}^{+0.003}$ & $1.110_{-0.005}^{+0.004}$ & - & $-0.816_{-0.006}^{+0.005}$ & $-0.816_{-0.006}^{+0.005}$ & - \\
& $1.127_{-0.001}^{+0.002}$ & $1.127_{-0.001}^{+0.002}$ & - & $-0.796_{-0.001}^{+0.003}$ & $-0.796_{-0.002}^{+0.002}$ & - \\
{$[11.0,12.5]$} & $1.198_{-0.005}^{+0.002}$ & $1.198_{-0.005}^{+0.002}$ & - & $-0.727_{-0.007}^{+0.005}$ & $-0.727_{-0.007}^{+0.005}$ & - \\
& $1.217_{-0.001}^{+0.001}$ & $1.217_{-0.001}^{+0.001}$ & - & $-0.698_{-0.001}^{+0.001}$ & $-0.698_{-0.001}^{+0.002}$ & - \\
{$[15.0,17.0]$} & $1.302_{-0.004}^{+0.002}$ & $1.302_{-0.004}^{+0.002}$ & $1.306_{-0.004}^{+0.002}$ & $-0.533_{-0.007}^{+0.005}$ & $-0.533_{-0.007}^{+0.005}$ & $-0.525_{-0.008}^{+0.006}$ \\
& $1.314_{-0.003}^{+0.002}$ & $1.314_{-0.003}^{+0.002}$ & $1.317_{-0.002}^{+0.003}$ & $-0.506_{-0.006}^{+0.005}$ & $-0.506_{-0.006}^{+0.005}$ & $-0.498_{-0.006}^{+0.006}$ \\
& $1.366_{-0.002}^{+0.001}$ & $1.366_{-0.002}^{+0.001}$ & $1.369_{-0.002}^{+0.001}$ & $-0.341_{-0.006}^{+0.004}$ & $-0.341_{-0.006}^{+0.004}$ & $-0.331_{-0.006}^{+0.004}$ \\
& $1.371_{-0.001}^{+0.001}$ & $1.371_{-0.001}^{+0.001}$ & $1.374_{-0.002}^{+0.001}$ & $-0.323_{-0.006}^{+0.005}$ & $-0.323_{-0.006}^{+0.005}$ & $-0.314_{-0.006}^{+0.006}$ \\
\hline
\end{tabular}

small, in the range of $10^{-4}-10^{-2}$, and clearly consistent with the $\mathrm{LHCb}$ measurements in the six bins

(4) For the physical observables $A_{\mathrm{FB}}^{l}, P_{1,2,3}$, and $P_{4,5,6,8}$, the experimental measurements are still absent now; we think that the PQCD and "PQCD+Lattice" predictions for these physical observables will be tested in the near future LHCb and Belle-II experiments

\section{Data Availability}

All relevant data are from $\mathrm{LHCb}$ Collaboration as given in References [31, 33].

\section{Conflicts of Interest}

The authors declare that they have no conflicts of interest.

\section{Acknowledgments}

This work (The preprint number of this paper is arXiv: 2011.11409 with the web link: https://arxiv.org/abs/2011 .11409) was supported by the National Natural Science Foundation of China under (Grant Nos. 11775117 and 11235005).

\section{References}

[1] LHCb Collaboration, "Lepton flavor universality tests in $b \rightarrow s l^{+} l^{-}$decays at LHCb," PoS ICHEP, vol. 69, 2018.

[2] LHCb Collaboration, "Test of lepton universality using $B^{+} \rightarrow K^{+} l^{+} l^{-}$decays," Physical Review Letters, vol. 113, article 151601, 2014.
[3] LHCb Collaboration, "Test of lepton universality with $B^{0}$ $\longrightarrow K^{* 0} l^{+} l^{-}$decays," Journal of High Energy Physics, vol. 1708, p. 55, 2017.

[4] M. Bordone, G. Isidori, and A. Pattori, "On the standard model predictions for $R_{K}$ and $R_{K^{*}}$," The European Physical Journal C, vol. 76, no. 8, p. 440, 2016.

[5] G. Hiller and F. Kruger, "More model-independent analysis of $b \rightarrow s$ processes," Physical Review D, vol. 69, no. 7, article 074020, 2004.

[6] G. Hiller and M. Schmaltz, "Diagnosing lepton-nonuniversality in $b \longrightarrow$ sle," Journal of High Energy Physics, vol. 2015, no. 2, p. 55, 2015.

[7] S. Descotes-Genon, J. Matias, and J. Virto, "Understanding the $B \longrightarrow K^{*} \mu^{+} \mu^{-}$anomaly," Physical Review D, vol. 88, no. 7, article 074002, 2013.

[8] J. Matias, F. Mescia, M. Ramon, and J. Virto, "Complete anatomy of $\bar{B}_{d} \longrightarrow \bar{K}^{* 0}(\longrightarrow K \pi) l^{+} l^{-}$and its angular distribution," Journal of High Energy Physics, vol. 2012, no. 4, p. 104, 2012.

[9] J. Matias and N. Serra, "Symmetry relations between angular observables in $B^{0} \longrightarrow K^{*} \mu^{+} \mu^{-}$and the LHCb $P_{5}^{\prime}$ anomaly," Physical Review D, vol. 90, no. 3, article 034002, 2014.

[10] S. Descotes-Genon, J. Matias, M. Ramon, and J. Virto, "Implications from clean observables for the binned analysis of $B$ $\longrightarrow K^{*} \mu^{+} \mu^{-}$at large recoil," Journal of High Energy Physics, vol. 2013, no. 1, p. 48, 2013.

[11] S. Descotes-Genon, T. Hurth, J. Matias, and J. Virto, "Optimizing the basis of $B \longrightarrow K^{*} l l$ observables in the full kinematic range," Journal of High Energy Physics, vol. 2013, no. 5, p. 137, 2013.

[12] LHCb Collaboration, "Measurement of form-factorindependent observables in the decay $B^{0} \longrightarrow K^{*} \mu^{+} \mu^{-}$," Physical Review Letter, vol. 111, article 191801, 2013.

[13] LHCb Collaboration, "Angular analysis of the $B^{0} \longrightarrow K^{*} \mu^{+} \mu^{-}$ decay using $3 \mathrm{fb}^{-1}$ of integrated luminosity," Journal of High Energy Physics, vol. 1602, p. 104, 2016. 
[14] Belle Collaboration, "Angular analysis of $B^{0} \longrightarrow K^{*}(892)^{0} \ell^{+} \ell^{-}$ ," https://arxiv.org/abs/1604.04042.

[15] S. Descotes-Genon, L. Hofer, J. Matias, and J. Virto, "On the impact of power corrections in the prediction of $B \longrightarrow K^{*} \mu^{+}$ $\mu^{-}$observables," Journal of High Energy Physics, vol. 2014, no. 12, p. 125, 2014.

[16] A. Deandrea and A. D. Polosa, "The exclusive $B_{s} \rightarrow \phi \mu^{+} \mu^{-}$process in a constituent quark model," Physical Review D, vol. 64, no. 7, article 074012, 2001.

[17] S. Dubnicka, A. Z. Dubnickova, A. Issadykov, M. A. Ivanov, A. Liptaj, and S. K. Sakhiyev, "Decay $B_{s} \longrightarrow \phi \ell^{+} \ell^{-}$in covariant quark model," Physical Review D, vol. 93, no. 9, article 094022, 2016.

[18] C. Q. Geng and C. C. Liu, "Study of $B_{s} \longrightarrow\left(\eta, \eta^{\prime}, \phi\right) \ell \bar{\ell}$ decays," Journal of Physics G: Nuclear and Particle Physics, vol. 29, no. 6, pp. 1103-1117, 2003.

[19] C. Bobeth, G. Hiller, and G. Piranishvili, " $\mathrm{CP}$ asymmetries in $\bar{B} \longrightarrow \bar{K}^{*}(\longrightarrow \bar{K} \pi) \bar{\ell} \ell$ and untagged $\bar{B}_{s}, B_{s} \longrightarrow \phi\left(\longrightarrow K^{+} K^{-}\right) \bar{\ell} \ell$ decays at NLO," Journal of High Energy Physics, vol. 2008, no. 7, p. 106, 2008.

[20] P. Ball and R. Zwicky, " $B_{d, s} \rightarrow \rho, \omega, K^{*}, \phi$ decay form-factors from light-cone sum rules revisited," Physical Review D, vol. 71, no. 1, article 014029, 2005.

[21] Y. L. Wu, M. Zhong, and Y. B. Zuo, " $B_{s} D_{s} \longrightarrow \pi, K, \eta, \rho, K^{*}, \omega, \phi$ transition form factors and decay rates with extraction of the CKM parameters $\left|V_{u b}\right|,\left|V_{c s}\right|,\left|V_{c d}\right|$," International Journal of Modern Physics A, vol. 21, no. 30, pp. 6125-6172, 2006.

[22] W. Altmannshofer and D. M. Straub, "New physics in $b \rightarrow s$ transitions after LHC run I," The European Physical Journal C, vol. 75, no. 8, p. 382, 2015.

[23] A. Bharucha, D. M. Straub, and R. Zwicky, " $B \longrightarrow V \ell^{+} \ell^{-}$in the standard model from light-cone sum rules," Journal of High Energy Physics, vol. 2016, no. 8, p. 98, 2016.

[24] J. Gao, C. D. Lü, Y. L. Shen, Y. M. Wang, and Y. B. Wei, "Precision calculations of $B \rightarrow V$ form factors from soft-collinear effective theory sum rules on the light-cone," Physical Review $D$, vol. 101, no. 7, article 074035, 2020.

[25] S. Descotes-Genon, L. Hofer, J. Matias, and J. Virto, "Global analysis of $b \longrightarrow$ sll anomalies," Journal of High Energy Physics, vol. 2016, no. 6, p. 92, 2016.

[26] R. Mohanta and A. K. Giri, "Study of FCNC mediated rare $B_{s}$ decays in a single universal extra dimension scenario," Physical Review D, vol. 75, no. 3, article 035008, 2007.

[27] Y. Li and J. Hua, "Study of $B_{s} \longrightarrow \phi \ell^{+} \ell^{-}$decay in a single universal extra dimension," The European Physical Journal $C$, vol. 71, no. 9, article 1764, 2011.

[28] Y. G. Xu, L. H. Zhou, B. Z. Li, and R. M. Wang, "Analysis of $B_{s} \rightarrow \phi \mu^{+} \mu^{-}$decay within supersymmetry," Chinese Physics $C$, vol. 37, no. 6, article 063104, 2013.

[29] CDF Collaboration, "Measurement of the forward-backward asymmetry in the $B \longrightarrow K^{(*)} \mu^{+} \mu^{-}$decay and first observation of the $B_{s}^{0} \longrightarrow \phi \mu^{+} \mu^{-}$decay," Physical Review Letter, vol. 106, article 161801, 2011.

[30] LHCb Collaboration, "Differential branching fraction and angular analysis of the decay $B_{s}^{0} \longrightarrow \phi \mu^{+} \mu^{-}$," Journal of High Energy Physics, vol. 1307, p. 84, 2013.

[31] LHCb Collaboration, "Angular analysis and differential branching fraction of the decay $B_{s}^{0} \longrightarrow \phi \mu^{+} \mu^{-}$," Journal of High Energy Physics, vol. 1509, p. 179, 2015.
[32] LHCb Collaboration, "Precise measurement of the $f_{s} / f_{d}$ ratio of fragmentation fractions and of $B_{s}^{0}$ decay branching fractions," Physical Review D, vol. 104, article 032005, 2021.

[33] LHCb Collaboration, "Branching fraction measurements of the rare $B_{s}^{0} \longrightarrow \phi \mu^{+} \mu^{-}$and $B_{s}^{0} \longrightarrow f_{2}^{\prime}(1525) \mu^{+} \mu^{-}$decays," https://arxiv.org/abs/2105.14007.

[34] LHCb Collaboration, "Angular analysis of the rare decay $B_{s}^{0}$ $\longrightarrow \phi \mu^{+} \mu^{-}$, " https://arxiv.org/abs/2107.13428.

[35] S. P. Jin, X. Q. Hu, and Z. J. Xiao, "Study of $B_{s} \longrightarrow K^{(*)} \ell^{+} \ell^{-}$ decays in the PQCD factorization approach with lattice QCD input," Physical Review D, vol. 102, no. 1, article 013001, 2020.

[36] Y. Y. Keum, H. N. Li, and A. I. Sanda, "Penguin enhancement and $B \longrightarrow K \pi$ decays in perturbative QCD," Physical Review $D$, vol. 63 , no. 5 , article $054008,2001$.

[37] C. D. Lu, K. Ukai, and M. Z. Yang, "Branching ratio and CP violation of $B \longrightarrow \pi \pi$ decays in perturbative QCD approach," Physical Review D, vol. 63, no. 7, article 074009, 2001.

[38] H. N. Li, "QCD aspects of exclusive B meson decays," Progress in Particle and Nuclear Physic, vol. 51, no. 1, pp. 85-171, 2003.

[39] H. N. Li and S. Mishima, "Pion transition form factor in $k_{T}$ factorization," Physical Review D, vol. 80, no. 7, article 074024, 2009.

[40] Y. Y. Fan, W. F. Wang, S. Cheng, and Z. J. Xiao, "Anatomy of $B \longrightarrow K \eta^{(\prime)}$ decays in different mixing schemes and effects of next-to-leading order contributions in the perturbative QCD approach," Physical Review D, vol. 87, no. 9, article 094003, 2013.

[41] Y. Y. Fan, W. F. Wang, S. Cheng, and Z. J. Xiao, "Semileptonic decays $B \longrightarrow D^{(*)} l v$ in the perturbative QCD factorization approach," Chinese Science Bulletin, vol. 59, no. 2, pp. 125132, 2014.

[42] A. Ali, G. Kramer, Y. Li et al., "Charmless non-leptonic $B_{s}$ decays to $P P, P V$ and $V V$ final states in the pQCD approach," Physical Review D, vol. 76, no. 7, article 074018, 2007.

[43] Z. J. Xiao, W. F. Wang, and Y. Y. Fan, "Revisiting the pure annihilation decays $B_{s} \longrightarrow \pi^{+} \pi^{-}$and $B^{0} \longrightarrow K^{+} K^{-}$: the data and the pQCD predictions," Physical Review D, vol. 85, no. 9, article 094003, 2012.

[44] W. F. Wang and Z. J. Xiao, "The semileptonic decays $B / B_{s}$ $\longrightarrow(\pi, K)\left(l^{+} l^{-}, l v, v \bar{v}\right)$ in the perturbative QCD approach beyond the leading-order," Physical Review D, vol. 86, no. 11, article 114025, 2012.

[45] W. F. Wang, Y. Y. Fan, M. Liu, and Z. J. Xiao, "Semileptonic decays $B / B_{s} \longrightarrow\left(\eta, \eta^{\prime}, G\right)\left(l^{+} l^{-}, l \bar{v}, v \bar{v}\right)$ in the perturbative QCD approach beyond the leading order," Physical Review $D$, vol. 87, no. 9, article 097501, 2013.

[46] D. C. Yan, P. Yang, X. Liu, and Z. J. Xiao, "Anatomy of $B_{s} \longrightarrow P V$ decays and effects of next-to-leading order contributions in the perturbative QCD factorization approach," Nuclear Physics B, vol. 931, pp. 79-104, 2018.

[47] D. C. Yan, X. Liu, and Z. J. Xiao, "Anatomy of $B_{s} \longrightarrow V V$ decays and effects of next-to-leading order contributions in the perturbative QCD factorization approach," Nuclear Physics B, vol. 935, pp. 17-39, 2018.

[48] M. Döring, U. G. Meißner, and W. Wang, "Chiral dynamics and S-wave contributions in semileptonic B decays," Journal of High Energy Physics, vol. 2013, no. 10, p. 11, 2013.

[49] T. Kurimoto, H. N. Li, and A. I. Sanda, "Leading power contributions to $B \longrightarrow \pi, \rho$ transition form-factors," Physical Review $D$, vol. 65, article 014007, 2001. 
[50] C. D. Lu and M. Z. Yang, " $B$ to light meson transition form factors calculated in perturbative QCD approach," The European Physical Journal C, vol. 28, no. 4, pp. 515-523, 2003.

[51] P. Ball, V. M. Braun, and A. Lenz, "Higher-twist distribution amplitudes of the $K$ meson in QCD," Journal of High Energy Physics, vol. 2006, no. 5, p. 4, 2006.

[52] G. Buchalla, A. J. Buras, and M. E. Lautenbacher, "Weak decays beyond leading logarithms,", Reviews of Modern Physics, vol. 68, no. 4, pp. 1125-1244, 1996.

[53] R. H. Li, C. D. Lu, W. Wang, and X. X. Wang, " $B \rightarrow S$ transition form factors in the PQCD approach," Physical Review D, vol. 79, no. 1, article 014013, 2009.

[54] S. R. Singh and B. Mawlong, " $331-Z$ ' mediated FCNC effects on $b \rightarrow d \mu^{+} \mu^{-}$transitions," International Journal of Modern Physics A, vol. 33, article 1850225, 2018.

[55] B. Kindra and N. Mahajan, "Predictions of angular observables for $\bar{B}_{s} \longrightarrow K^{*} \ell \ell$ and $\bar{B} \longrightarrow \rho \ell \ell$ in the standard model," Physical Review D, vol. 98, no. 9, article 094012, 2018.

[56] P. Nayek, P. Maji, and S. Sahoo, "Study of semileptonic decays $B \rightarrow \pi l^{+} I^{-}$and $B \rightarrow p l^{+} l^{-}$in nonuniversal $Z^{\prime}$ model," Physical Review D, vol. 99, no. 1, article 013005, 2019.

[57] K. G. Chetyrkin, M. Misiak, and M. Münz, "Weak radiative Bmeson decay beyond leading logarithms," Physics Letters $B$, vol. 400, no. 1-2, pp. 206-219, 1997.

[58] K. G. Chetyrkin, M. Misiak, and M. Munz, “ $|\Delta F|=1$ nonleptonic effective Hamiltonian in a simpler scheme," Nuclear Physics B, vol. 520, no. 1-2, pp. 279-297, 1998.

[59] P. Gambino, M. Gorbahn, and U. Haisch, "Anomalous dimension matrix for radiative and rare semileptonic $B$ decays up to three loops," Nuclear Physics B, vol. 673, no. 1-2, pp. 238-262, 2003.

[60] C. Bobeth, M. Misiak, and J. Urban, "Photonic penguins at two loops and $m_{t}$ dependence of $B R\left(B \rightarrow X_{s} l^{+} l^{-}\right)$," Nuclear Physics $B$, vol. 574, no. 1-2, pp. 291-330, 2000.

[61] C. H. Chen and C. Q. Geng, "Baryonic rare decays of $\Lambda(b) \rightarrow \Lambda l^{+} l^{-}$," Physical Review D, vol. 64, no. 7, article 074001, 2001.

[62] C. S. Lim, T. Morozumi, and A. I. Sanda, "A prediction for $d$ $\Gamma(b \longrightarrow s \ell \bar{\ell}) / d q^{2}$ including the long distance effects," Physics Letters B, vol. 218, no. 3, pp. 343-347, 1989.

[63] N. G. Deshpande, J. Trampetic, and K. Panose, "Resonance background to the decays $b \longrightarrow s \ell^{+} \ell^{-}, B \longrightarrow K^{*} \ell^{+} \ell^{-}$and $B$ $\longrightarrow K \ell^{+} \ell^{-}$, , Physical Review D, vol. 39, no. 5, pp. 1461$1463,1989$.

[64] A. Ali, T. Mannel, and T. Morozumi, "Forward backward asymmetry of dilepton angular distribution in the decay $b$ $\longrightarrow s \ell^{+} \ell^{-}$, , Physics Letters B, vol. 273, no. 4, pp. 505-512, 1991.

[65] P. J. O'Donnell and H. K. K. Tung, "Resonance contributions to the decay $b \longrightarrow s \ell^{+} \ell^{-}$," Physical Review D, vol. 43, no. 7, article 2067, pp. R2067-R2069, 1991.

[66] A. Khodjamirian, T. Mannel, A. A. Pivovarov, and Y. M. Wang, "Charm-loop effect in $B \longrightarrow K^{(*)} l^{+} l^{-}$and $B \longrightarrow K^{*}$ $\gamma$," Journal of High Energy Physics, vol. 2010, no. 9, p. 89, 2010.

[67] A. Khodjamirian, T. Mannel, Y. M. Wang, and Y. M. Wang, " $B \rightarrow K l^{+} l^{-}$decay at large hadronic recoil," Journal of High Energy Physics, vol. 2013, no. 2, p. 10, 2013.

[68] I. Ahmed, M. J. Aslam, and M. A. Paracha, "Impact of $Z^{\prime}$ and universal extra dimension parameters on different asymme- tries in $B_{s} \longrightarrow \phi \ell^{+} \ell^{-}$decays," Physical Review D, vol. 88, no. 1 , article $014019,2013$.

[69] W. F. Wang, H. N. Li, W. Wang, and C. D. Lu, " $S$-wave resonance contributions to the $B_{(s)}^{0} \longrightarrow J / \psi \pi^{+} \pi^{-}$and $B_{s} \rightarrow \pi^{+} \pi^{-} \mu^{+} \mu^{-}$decays," Physical Review D, vol. 91, no. 9, article $094024,2015$.

[70] W. Altmannshofer, P. Ball, A. Bharucha, A. J. Buras, D. M. Straub, and M. Wick, "Symmetries and asymmetries of $B$ $\longrightarrow K^{*} \mu^{+} \mu^{-}$decays in the standard model and beyond," Journal of High Energy Physics, vol. 2009, no. 1, p. 19, 2009.

[71] D. Becirevic and E. Schneider, "On transverse asymmetries in $B \longrightarrow K^{*} l^{+} l^{-}$," Nuclear Physics B, vol. 854, no. 2, pp. 321-339, 2012.

[72] U. Egede, T. Hurth, J. Matias, M. Ramon, and W. Reece, "New physics reach of the decay mode $\bar{B} \longrightarrow \bar{K}^{* 0} \ell^{+} \ell^{-}$," Journal of High Energy Physics, vol. 2010, no. 10, p. 56, 2010.

[73] U. Egede, T. Hurth, J. Matias, M. Ramon, and W. Reece, "New observables in the decay mode $\bar{B}_{d} \longrightarrow \bar{K}^{* 0} \ell^{+} \ell^{-}$," Journal of High Energy Physics, vol. 2008, no. 11, p. 32, 2008.

[74] Particle Data Group, "Review of particle physics," Physical Review D, vol. 98, article 030001, 2018.

[75] Y. M. Wang and Y. L. Shen, "QCD corrections to $B \longrightarrow \pi$ form factors from light-cone sum rules," Nuclear Physics B, vol. 898, pp. 563-604, 2015.

[76] F. Kruger and J. Matias, "Probing new physics via the transverse amplitudes of $B^{0} \longrightarrow K^{* 0}\left(\longrightarrow K^{+} \pi-\right) l^{+} l^{-}$at large recoil," Physical Review D, vol. 71, no. 9, article 094009, 2005.

[77] J. Gratrex, M. Hopfer, and R. Zwicky, "Generalised helicity formalism, higher moments and the $B \longrightarrow K_{J_{K}}(\longrightarrow K \pi) \bar{l}_{1} l_{2}$ angular distributions," Physical Review D, vol. 93, no. 5, article 054008, 2016.

[78] H. Y. Cheng, C. K. Chua, and C. W. Hwang, "Covariant lightfront approach fors-wave and p-wave mesons: its application to decay constants and form factors," Physical Review D, vol. 69, no. 7, article 074025, 2004.

[79] W. Wang, Y. L. Shen, and C. D. Lu, "Covariant light-front approach for $B_{c}$ transition form factors," Physical Review D, vol. 79, no. 5, article 054012, 2009.

[80] Y. Y. Fan, Z. J. Xiao, R. M. Wang, and B. Z. Li, “The $B \longrightarrow$ $D^{(*)} l v_{l}$ decays in the pQCD approach with the lattice QCD input," Science Bulletin, vol. 60, no. 23, pp. 2009-2015, 2015.

[81] X. Q. Hu, S. P. Jin, and Z. J. Xiao, "Semileptonic decays $B_{c}$ $\longrightarrow\left(\eta_{c}, J / \psi\right) l \bar{v}_{l}$ in the "PQCD + Lattice" approach," Chinese Physics C, vol. 44, no. 2, article 023104, 2020.

[82] X. Q. Hu, S. P. Jin, and Z. J. Xiao, "Semileptonic decays $B / B_{s}$ $\longrightarrow\left(D^{(*)}, D_{s}^{(*)}\right) l v_{l}$ in the PQCD factorization approach with the lattice QCD input," Chinese Physics C, vol. 44, no. 5, article 053102, 2020.

[83] C. Bourrely, L. Lellouch, and I. Caprini, "Model-independent description of $B \rightarrow \pi l v$ decays and a determination of $\left|V_{u b}\right|$," Physical Review D, vol. 79, no. 1, article 013008, 2009.

[84] D. Leljak, B. Melic, and M. Patra, "On lepton flavour universality in semileptonic $B_{c} \rightarrow \eta_{c}$ J/ $\Psi$ decays," Journal of High Energy Physics, vol. 2019, no. 5, p. 94, 2019.

[85] R. H. Li, C. D. Lu, and W. Wang, "Transition form factors of $B$ decays in top-wave axial-vector mesons in the perturbative QCD approach,” Physical Review D, vol. 79, no. 3, article 034014, 2009.

[86] U. O. Yilmaz, "Analysis of $B_{s} \longrightarrow \phi \ell^{+} \ell^{-}$decay with new physics effects," The European Physical Journal C, vol. 58, no. 4, pp. 555-568, 2008. 
[87] Y. Q. Peng and M. Z. Yang, "Study of semileptonic decay of $\bar{B}_{s}^{0} \longrightarrow \phi l^{+} l^{-}$in QCD sum rule," Communications in Theoretical Physics, vol. 72, no. 9, article 095201, 2020.

[88] D. Melikhov and B. Stech, "Weak form factors for heavy meson decays: an update," Physical Review D, vol. 62, no. 1, article 014006, 2000.

[89] R. N. Faustov and V. O. Galkin, "Rare $B_{s}$ decays in the relativistic quark model," The European Physical Journal C, vol. 73, no. 10, p. 2593, 2013.

[90] C. D. Lu, W. Wang, and Z. T. Wei, "Heavy-to-light form factors on the light cone," Physical Review D, vol. 76, no. 1, article 014013, 2007.

[91] F. Su, Y. L. Wu, Y. B. Yang, and C. Zhuang, "Charmless $B_{s} \rightarrow P P, P V, V V$ decays based on the six-quark effective Hamiltonian with strong phase effects II," The European Physical Journal C, vol. 72, no. 3, article 1914, 2012.

[92] R. R. Horgan, Z. Liu, S. Meinel, and M. Wingate, "Lattice QCD calculation of form factors describing the rare decays $B \longrightarrow$ $K^{*} \ell^{+} \ell^{-}$and $B_{s} \longrightarrow \phi \ell^{+} \ell^{-}$," Physical Review D, vol. 89, no. 9, article 094501, 2014.

[93] C. D. Lu, Y. L. Shen, Y. M. Wang, and Y. B. Wei, "QCD calculations of $B \rightarrow \pi, K$ form factors with higher-twist corrections," Journal of High Energy Physics, vol. 2019, no. 1, p. 24, 2019.

[94] A. Bharucha, T. Feldmann, and M. Wick, "Theoretical and phenomenological constraints on form factors for radiative and semi-leptonic B-meson decays," Journal of High Energy Physics, vol. 2010, no. 9, p. 90, 2010.

[95] D. Bigi and P. Gambino, "Revisiting $B \longrightarrow D \ell v$," Physical Review D, vol. 94, no. 9, article 094008, 2016.

[96] BaBar Collaboration, "Direct CP, lepton flavor and isospin asymmetries in the decays $B \longrightarrow K^{(*)} \ell^{+} \ell^{-}$," Physical Review Letter, vol. 102, article 091803, 2009.

[97] S. Descotes-Genon and J. Virto, "Time dependence in $B \rightarrow \mathrm{Vll}$ decays," Journal of High Energy Physics, vol. 2015, no. 7, p. 45, 2015. 